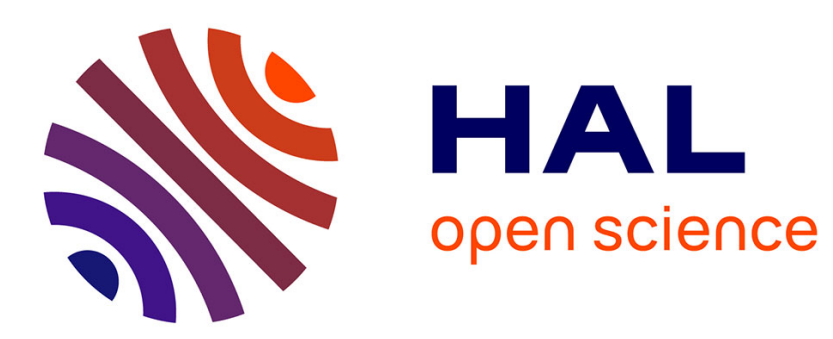

\title{
Accurate Asymptotic Preserving Boundary Conditions for Kinetic Equations on Cartesian Grids
}

Florian Bernard, Angelo Iollo, Gabriella Puppo

\section{To cite this version:}

Florian Bernard, Angelo Iollo, Gabriella Puppo. Accurate Asymptotic Preserving Boundary Conditions for Kinetic Equations on Cartesian Grids. Journal of Scientific Computing, 2015, pp.34. 10.1007/s10915-015-9984-8 . hal-01148397

\section{HAL Id: hal-01148397 \\ https://inria.hal.science/hal-01148397}

Submitted on 4 May 2015

HAL is a multi-disciplinary open access archive for the deposit and dissemination of scientific research documents, whether they are published or not. The documents may come from teaching and research institutions in France or abroad, or from public or private research centers.
L'archive ouverte pluridisciplinaire HAL, est destinée au dépôt et à la diffusion de documents scientifiques de niveau recherche, publiés ou non, émanant des établissements d'enseignement et de recherche français ou étrangers, des laboratoires publics ou privés. 


\title{
Accurate Asymptotic Preserving Boundary Conditions for Kinetic Equations on Cartesian Grids
}

\author{
Florian BERNARD ${ }^{1,2}$, Angelo IOLLO ${ }^{2}$, and Gabriella PUPPO ${ }^{3}$ \\ ${ }^{1}$ Department of Mechanical and Aerospace Engineering, Politecnico di Torino, Italy. \\ ${ }^{2}$ Univ. Bordeaux, IMB, UMR 5251, F-33400 Talence, France., INRIA, F-33400 Talence, France. \\ ${ }^{3}$ Dip. di Scienza ed Alta Tecnologia, Università dell'Insubria, Como, Italy.
}

May 4, 2015

\begin{abstract}
A simple second-order scheme on Cartesian grids for kinetic equations is presented, with emphasis on the accurate enforcement of wall boundary conditions on immersed bodies. This approach preserves at the discrete level the asymptotic limit towards Euler equations up to the wall, thus ensuring a smooth transition towards the hydrodynamic regime. We investigate exact, numerical and experimental test cases for the BGK model in order to assess the accuracy of the method.
\end{abstract}

\section{Introduction}

A typical feature of complex gas flows is the presence of both continuum and rarefied regimes in the same field. The Boltzmann equation is the main tool for modelling rarefied gas regimes [8]:

$$
\frac{\partial f}{\partial t}(\mathbf{x}, \boldsymbol{\xi}, t)+\boldsymbol{\xi} \cdot \nabla_{\mathbf{x}} f(\mathbf{x}, \boldsymbol{\xi}, t)=Q(f, f)
$$

where $f$ is the distribution function with an initial data given by $f_{0}(\mathbf{x}, \boldsymbol{\xi}, t=0)$, $\mathbf{x}$ is the space variable of dimension $d, \boldsymbol{\xi}$ is the microscopic velocity variable of dimension $N$ and $Q(f, f)$ is a bilinear operator that models the collisions between particles.

Rarefied flow regimes are characterized by a high Knudsen number defined as follows:

$$
K n=\frac{\lambda}{L}
$$

where $\lambda$ is the mean free path of the particles and $\mathrm{L}$ is the characteristic length of the problem. When the Knudsen number goes to zero, the continuum regime is approached. In this regime the flow can be modelled thanks to Navier-Stokes equations for small Knudsen numbers, or Euler equations when $K n \rightarrow 0$. For Knudsen numbers greater than $10^{-1}$ (Knudsen number cut off) it is well known that these macroscopic models cannot accurately describe the flow and Boltzmann equation has to be used [6], [8]. Also, the local behaviour of the flow should be taken into account to determine the correct regime. For exemple, the presence of shocks or boundaries may result in kinetic flow also when the Knudsen number is small. Thus, the Knudsen number cut off is just an estimate of the breakdown of macroscopic models but it is not an absolute threshold. 
The main fields of application of Boltzmann equation are those where the mean free path becomes large compared to the scales of the phenomenon under consideration. This is typically the case for space re-entry problems where the density dramatically decreases in the upper layers of the planetary atmosphere. More recently, applications are also found in nano systems, even in standard gas conditions, [27].

Directly solving the Boltzmann equation is computationally prohibitive because of highdimensionality and of the complexity of the collision operator $Q(f, f)$, see for instance [13]. However, several numerical models exist to get around this difficulty.

One of the most popular model is the Direct Simulation Monte Carlo (DSMC, [6]). This is a statistical approximation of the Boltzmann equation that is numerically viable for high Knudsen numbers, but becomes very costly for low Knudsen numbers. For these regimes it also has the disadvantage of generating noisy results.

The BGK equation [5] is a computationally efficient approximation of the Boltzmann equation for kinetic regimes at relatively low Knudsen numbers. It is based on a simple model for the collision term, that is expressed as a relaxation of the distribution function towards an equilibrium Maxwellian distribution. It is solved with a discrete velocity approach (DVM) and it is less costly than DSMC or direct solvers in hydrodynamic regimes. See [4] for additional work on computational time reduction. In this work, we will consider the BGK equation as kinetic model, but the structure of the boundary condition we propose can be extended to more general kinetic models.

The BGK model is by construction consistent with the hydrodynamic limit at the continuous level, i.e., for very small Knudsen numbers the Euler limit is recovered. The numerical schemes used to solve the BGK model that respect this property are called asymptotic preserving (AP), after the pioneering work [25]. Many schemes available today can be proven to be AP, see for instance the deterministic methods of [34]; the Exponential Runge Kutta schemes of [14]; the Micro-Macro decomposition of [3], the fully implicit scheme in [37], or the Unified Gas-kinetic scheme of [41]. See also the review in [26]. However the same attention has not been devoted to the AP enforcement of boundary conditions. Also the task of preserving accuracy up to the wall is not trivial [18], and moreover the behaviour of kinetic boundary conditions, as the Knudsen number approaches zero, to our knowledge has not yet been dealt with. In this work we show that a naive implementation of standard kinetic boundary conditions creates a spurious energy flux at the boundary, which is inconsistent with the Euler set up. The AP boundary condition proposed in this work will also be relevant when rarefied fluid flow is approached using domain decomposition techniques, see for instance $[40,12,1]$, where it is necessary to ensure a smooth transition between the kinetic regime and the hydrodynamic regime. We propose a simple modification that ensures that the scheme remains AP up to the boundary. In this sense, two contributions are present in this paper. We start from the consideration that standard specular reflection at solid walls is a consistent asymptotic preserving boundary condition at the continuous level. However, we show that at the discrete level specular reflection introduces spurious boundary layers also in the limit of very small Knudsen numbers. Therefore, we first consider the problem of devising a numerically consistent boundary condition of the kinetic scheme in the hydrodynamic regime. Next, we investigate a second-order accurate method to enforce this boundary condition on solid wall arbitrarily crossing a Cartesian mesh.

The use of Cartesian grids is motivated by the fact that they allow a drastic reduction of the computational setup for complex unsteady geometries. For body-fitted grids, in the case of moving geometries, at each time step a new mesh has to be generated. Accurate moving mesh techniques have been recently developed for diluted gas flows [24], [9]. Nonetheless, body-fitted schemes for moving and possibly deforming multiple bodies evolving in a rarefied flow do not seem a computationally viable approach in general. The schemes resulting from Cartesian meth- 
ods are easily parallelizable and they can efficiently be mapped to high-performance computer architectures. They avoid dealing with grid generation and grid adaptation, a prohibitive task when the boundaries are moving. However, in order to preserve adequate spatial accuracy at the boundaries arbitrarely crossing, the effort is transferred to the enforcement of the boundary condition near the body.

Peskin [35] first introduced the immersed boundary method (IBM) to study biological flow problems governed by the incompressible Navier-Stokes equations. The idea was to add singular forcing terms in the momentum equation to take into account the effect exerted by the solid on the fluid. Another class of immersed boundary methods is the so-called Cartesian cut-cell methods [42], [22]. In this approach, the boundary cells are cut to fit the body. This may lead to singular cells that are not square any more but polygons. If the size of the polygons becomes too small or if a polygon is too deformed, a heavy restriction is imposed on the time step and accuracy. To avoid this, problematic cells are merged to neighbours and new cells are created, but this task is still a delicate issue in general geometries. Another possibility is to introduce ghost cells in the solid where fictitious data are computed in order to enforce boundary conditions at cells interfaces [15], [16]. Then the fluxes are modified to take into account the presence of the body. This idea has already been developed to second-order accuracy for Euler equations [21] and elliptic equations [11], [19]. In the present work it is extended to the BGK equation with a special care on preserving the asymptotic limit towards Euler equations. Even if most test cases are presented near the hydrodynamic regime, because as we show it is in this regime that standard boundary conditions fail, it is necessary to keep in mind a more general framework where different regimes are present. To illustrate this point, the last test cases will show the necessity of preserving the asymptotic properties of kinetic models up to the boundaries, to avoid spurious results.

\section{Governing equations}

In the following we will present in some detail the model equations and the discrete integration scheme for sake of completeness and to fix notation.

\subsection{The dimensionless BGK model}

The BGK model gives a simple representation of the collision term $Q(f, f)$ :

$$
\frac{\partial f}{\partial t}(\mathbf{x}, \boldsymbol{\xi}, t)+\boldsymbol{\xi} \cdot \nabla_{\mathbf{x}} f(\mathbf{x}, \boldsymbol{\xi}, t)=\frac{1}{\tau}\left(M_{f}(\mathbf{x}, \boldsymbol{\xi}, t)-f(\mathbf{x}, \boldsymbol{\xi}, t)\right)
$$

where $\mathbf{x}=(x, y, z)^{T}, \boldsymbol{\xi}=\left(\xi_{u}, \xi_{v}, \xi_{w}\right)^{T}$. In a more general case, $N$ denotes the number of dimensions in velocity space. The relaxation time is $\tau$ and $M_{f}$ is the Maxwellian distribution function, that is obtained as follows:

$$
M_{f}(\mathbf{x}, \boldsymbol{\xi}, t)=\frac{\rho(\mathbf{x}, t)}{(2 \pi R T(\mathbf{x}, t))^{N / 2}} \exp \left(-\frac{|\boldsymbol{\xi}-\mathbf{U}(\mathbf{x}, t)|^{2}}{2 R T(\mathbf{x}, t)}\right)
$$

where $R$ is the specific gas constant, $T(\mathbf{x}, t), \mathbf{U}(\mathbf{x}, t)=(u, v, w)^{T}$ and $\rho(\mathbf{x}, t)$ are the macroscopic values of temperature, velocity and density, respectively. Macroscopic quantities are calculated from the moments of $f$ defined by:

$$
\left(\begin{array}{c}
\rho(\mathbf{x}, t) \\
\rho(\mathbf{x}, t) \mathbf{U}(\mathbf{x}, t) \\
E(\mathbf{x}, t)
\end{array}\right)=\int_{\mathbb{R}^{N}} f(\mathbf{x}, \boldsymbol{\xi}, t) \mathbf{m}(\boldsymbol{\xi}) d \boldsymbol{\xi} \quad \text { with } \quad \mathbf{m}(\boldsymbol{\xi})=\left(\begin{array}{c}
1 \\
\boldsymbol{\xi} \\
\frac{1}{2}|\boldsymbol{\xi}|^{2}
\end{array}\right)
$$


Here $E$ is the total energy obtained as follows:

$$
E(\mathbf{x}, t)=\frac{N}{2} \rho R T(\mathbf{x}, t)+\frac{1}{2} \rho|\mathbf{U}(\mathbf{x}, t)|^{2}
$$

We consider a monoatomic gas for which the ratio of specific heats $\gamma$ can be calculated as:

$$
\gamma=1+\frac{2}{N}
$$

In the following, $N=3$ and therefore $\gamma=5 / 3$.

The relaxation time for the BGK model can be written as:

$$
\tau^{-1}=c \rho T^{1-\nu} \quad \text { with } \quad c=\frac{R T_{0}^{\nu}}{\mu_{0}}
$$

where $\nu$ is the exponent of the viscosity law of the gas, $\mu_{0}$ is the reference viscosity of the gas at the reference temperature $T_{0}$.

Let the following dimensionless parameters:

$$
\begin{array}{llll}
\hat{t}=\frac{t}{t_{0}} & \hat{\mathbf{x}}=\frac{\mathbf{x}}{L} & \hat{\boldsymbol{\xi}}=\frac{\boldsymbol{\xi}}{\left(R T_{0}\right)^{1 / 2}} & \hat{\mathbf{U}}=\frac{\mathbf{U}}{\left(R T_{0}\right)^{1 / 2}} \\
\hat{\rho}=\frac{\rho}{\rho_{0}} & \hat{T}=\frac{T}{T_{0}} & \hat{f}=\frac{f}{\rho_{0} /\left(R T_{0}\right)^{N / 2}} & \hat{M}_{f}=\frac{\hat{\rho}}{(2 \pi \hat{T})^{N / 2}} \exp \left(-\frac{|\hat{\boldsymbol{\xi}}-\hat{\mathbf{U}}|^{2}}{2 \hat{T}}\right)
\end{array}
$$

then, the dimensionless form of the BGK equation is (hats are dropped for simplicity):

$$
S h \partial_{t} f+\boldsymbol{\xi} \cdot \nabla_{\mathbf{x}} f=\frac{1}{K n_{\infty}} \rho T^{1-\nu}\left(M_{f}-f\right)
$$

with

$$
S h=\frac{L}{\left(R T_{0}\right)^{1 / 2} t_{0}} \quad K n_{\infty}=\frac{\lambda}{L} \quad \text { with } \quad \lambda=\frac{\mu_{0}}{\sqrt{R T_{0}} \rho_{0}}
$$

where $S h$ is the so-called Strouhal number usually set to 1 by choosing an adapted time scale and $K n_{\infty}$ is the Knudsen number in the reference conditions.

In the following it will be useful to define a local Knudsen number that corresponds to the relaxation time in dimensionless form:

$$
\frac{1}{\tau}=\frac{1}{K n_{\text {local }}}=\frac{1}{K n_{\infty}} \rho T^{1-\nu}
$$

\subsection{BGK reduced model}

Let us consider the dimensionless BGK model with $S h=1$. Thanks to the Chu reduction [10], in $1 \mathrm{D}$ and $2 \mathrm{D}$ it is possible to reduce the number of independent variables in velocity space. Note that this reduction is not an approximation but is exact. For the 1D case, let:

$$
\begin{aligned}
\phi\left(x, \xi_{u}, t\right) & =\int_{\mathbb{R}^{2}} f(x, \boldsymbol{\xi}, t) d \xi_{v} d \xi_{w} \\
\psi\left(x, \xi_{u}, t\right) & =\int_{\mathbb{R}^{2}} \frac{1}{2}\left(\xi_{v}^{2}+\xi_{w}^{2}\right) f(x, \boldsymbol{\xi}, t) d \xi_{v} d \xi_{w}
\end{aligned}
$$


and multiply the full model by $\mathbf{m}_{1}(\boldsymbol{\xi})=\left(1, \xi_{u}, \frac{1}{2} \xi_{u}^{2}\right)^{T}$. After integration with respect to $\xi_{v}$ and $\xi_{w}$, we have:

$$
\left\{\begin{array}{l}
\partial_{t} \phi\left(x, \xi_{u}, t\right)+\xi_{u} \partial_{x} \phi\left(x, \xi_{u}, t\right)=\frac{1}{\tau}\left(M_{\phi}\left(x, \xi_{u}, t\right)-\phi\left(x, \xi_{u}, t\right)\right) \\
\partial_{t} \psi\left(x, \xi_{u}, t\right)+\xi_{u} \partial_{x} \psi\left(x, \xi_{u}, t\right)=\frac{1}{\tau}\left(M_{\psi}\left(x, \xi_{u}, t\right)-\psi\left(x, \xi_{u}, t\right)\right)
\end{array}\right.
$$

where $M_{\phi}=\int_{\mathbb{R}^{2}} M_{f} d \xi_{v} d \xi_{w}$ and $M_{\psi}=\int_{\mathbb{R}^{2}} \frac{1}{2}\left(\xi_{v}^{2}+\xi_{w}^{2}\right) M_{f} d \xi_{v} d \xi_{w}$. In the continuous case, these expressions are:

$$
\left\{\begin{array}{l}
M_{\phi}\left(\xi_{u}\right)=\frac{\rho(x, t)}{\sqrt{2 \pi T(x, t)}} \exp \left(-\frac{\left(\xi_{u}-u(x, t)\right)^{2}}{2 T(x, t)}\right) \\
M_{\psi}\left(\xi_{u}\right)=\frac{(N-1) T(x, t)}{2} \frac{\rho(x, t)}{\sqrt{2 \pi T(x, t)}} \exp \left(-\frac{\left(\xi_{u}-u(x, t)\right)^{2}}{2 T(x, t)}\right)=\frac{(N-1) T(x, t)}{2} M_{\phi}
\end{array}\right.
$$

where $u(x, t)$ and $T(x, t)$ are the macroscopic velocity and macroscopic temperature, respectively.

The same procedure can be applied in $2 \mathrm{D}$, integrating over the third component of the velocity. We obtain:

$$
\begin{aligned}
\phi\left(x, y, \xi_{u}, \xi_{v}, t\right) & =\int_{\mathbb{R}^{2}} f(x, y, \boldsymbol{\xi}, t) d \xi_{w} \\
\psi\left(x, y, \xi_{u}, \xi_{v}, t\right) & =\int_{\mathbb{R}^{2}} \frac{1}{2} \xi_{w}^{2} f(x, y, \boldsymbol{\xi}, t) d \xi_{w}
\end{aligned}
$$

and then:

$$
\left\{\begin{array}{l}
\partial_{t} \phi\left(\mathbf{X}, \xi_{2}, t\right)+\boldsymbol{\xi}_{\mathbf{2}} \cdot \nabla \phi\left(\mathbf{X}, \boldsymbol{\xi}_{\mathbf{2}}, t\right)=\frac{1}{\tau}\left(M_{\phi}\left(\mathbf{X}, \boldsymbol{\xi}_{2}, t\right)-\phi\left(\mathbf{X}, \boldsymbol{\xi}_{2}, t\right)\right) \\
\partial_{t} \psi\left(\mathbf{X}, \boldsymbol{\xi}_{2}, t\right)+\boldsymbol{\xi}_{2} \cdot \nabla \psi\left(\mathbf{X}, \boldsymbol{\xi}_{2}, t\right)=\frac{1}{\tau}\left(M_{\psi}\left(\mathbf{X}, \boldsymbol{\xi}_{2}, t\right)-\psi\left(\mathbf{X}, \boldsymbol{\xi}_{2}, t\right)\right)
\end{array}\right.
$$

where $\mathbf{X}=(x, y), \boldsymbol{\xi}_{2}=\left(\xi_{u}, \xi_{v}\right)^{T}, M_{\phi}=\int_{\mathbb{R}^{2}} M_{f} d \xi_{w}$ and $M_{\psi}=\int_{\mathbb{R}^{2}} \frac{1}{2} \xi_{w}^{2} M_{f} d \xi_{w}$. In the continuous case, these expressions are:

$$
\left\{\begin{array}{l}
M_{\phi}=\frac{\rho(\mathbf{X}, t)}{2 \pi T(\mathbf{X}, t)} \exp \left(-\frac{\left|\boldsymbol{\xi}_{2}-\mathbf{U}_{2}(\mathbf{X}, t)\right|^{2}}{2 T(\mathbf{X}, t)}\right) \\
M_{\psi}=\frac{(N-2) T(\mathbf{X}, t)}{2} \frac{\rho(\mathbf{X}, t)}{2 \pi T(\mathbf{X}, t)} \exp \left(-\frac{\left|\boldsymbol{\xi}_{2}-\mathbf{U}_{2}(\mathbf{X}, t)\right|^{2}}{2 T(X, t)}\right)=\frac{(N-2) T(X, t)}{2} M_{\phi}
\end{array}\right.
$$

where $\mathbf{U}_{2}=(u, v)$.

\subsection{The discrete model in velocity space}

By construction, the distribution function $f$ and the Maxwellian distribution function $M_{f}$ satisfy:

$$
\int_{\mathbb{R}^{3}} M_{f} \mathbf{m}(\boldsymbol{\xi}) d \boldsymbol{\xi}=\left(\begin{array}{l}
\rho(\mathbf{x}, t) \\
\rho(\mathbf{x}, t) \mathbf{U}(\mathbf{x}, t) \\
E(\mathbf{x}, t)
\end{array}\right)=\int_{\mathbb{R}^{3}} f \mathbf{m}(\boldsymbol{\xi}) d \boldsymbol{\xi}
$$

This is an essential property to ensure conservation of mass, momentum and energy. 
In the discrete case, a grid must be introduced in velocity space and integrals are evaluated by quadrature. Let $\langle.,$.$\rangle denote the quadrature rule. Based on the work of Cabannes et al.$ [7] on entropic Maxwellian states, Mieussens proved in [31] that a discrete Maxwellian can be expressed as $\widetilde{M}_{f}=\exp (\boldsymbol{\alpha} \cdot \mathbf{m}(\boldsymbol{\xi}))$, such that:

$$
\left\langle\widetilde{M}_{f}(\boldsymbol{\xi}), \mathbf{m}(\boldsymbol{\xi})\right\rangle=\left(\begin{array}{l}
\rho \\
\rho \mathbf{U} \\
E
\end{array}\right)
$$

at the discrete level, where we have used the same symbol for the discrete moments of $f$, with a slight abuse of notation.

The discrete Maxwellian distribution function $\widetilde{M}_{f}$ can be computed as the solution of a non-linear system solved with a Newton-Raphson algorithm. The algorithm is detailed in the Appendix, also for the reduced models.

We use a uniform velocity grid symmetric with respect to 0 and such that $f$ is negligible outside the grid. Hence, the trapezoidal quadrature rule is used because it has spectral accuracy for smooth and periodic functions on a uniform grid. In 1D:

$$
\mathcal{G}_{v}=\left(\xi_{u}^{i}\right)_{i=-n . . n} \quad \text { with } \quad \xi_{u}^{i}=i \Delta \xi_{u}
$$

For multidimensional cases, the same discretization is independently performed in all directions.

\section{The numerical method}

The numerical method will be described for one equation of the $2 \mathrm{D}$ reduced model. The two equations of the reduced model have the same form. Hence we employ the same scheme for both equations (on two different distribution functions).

\subsection{The space discretization}

The physical space $\Omega$ is discretized on a Cartesian grid with $n \times m$ cells:

$$
\Omega=\bigcup_{\substack{i=1 . . n \\ j=1 . . m}} \Omega_{i, j}=\bigcup_{\substack{i=1 . . n \\ j=1 . . m}}\left[x_{i-1 / 2}, x_{i+1 / 2}\right] \times\left[y_{j-1 / 2}, y_{j+1 / 2}\right]
$$

such that $\left(x_{i}, y_{j}\right)$ are the coordinates of the center of cell $(i, j)$ and $\left(x_{i+1 / 2}, y_{j}\right)$ are the coordinates of the center of the interface between cells $(i, j)$ and $(i+1, j)$. On a space cell $\Omega_{i, j}=\left[x_{i}-\right.$ $\left.\frac{\Delta x}{2}, x_{i}+\frac{\Delta x}{2}\right] \times\left[y_{j}-\frac{\Delta y}{2}, y_{j}+\frac{\Delta y}{2}\right]$, eq.(9) is integrated with a finite volume method:

$$
\frac{\partial f_{i, j}}{\partial t}+\boldsymbol{\xi} \cdot \int_{\partial \Omega_{i, j}} f \mathbf{n}_{\partial \Omega_{i, j}} d \sigma=\frac{1}{\tau_{i, j}}\left(M_{f_{i, j}}-f_{i, j}\right)
$$

where $f_{i, j}=\frac{1}{\left|\Omega_{i, j}\right|} \int_{\Omega_{i, j}} f d x d y$ and $M_{f_{i, j}}=\frac{1}{\left|\Omega_{i, j}\right|} \int_{\Omega_{i, j}} \widetilde{M}_{f} d x d y$. Here, $\sigma$ is the integration variable representing a surface element.

Since a uniform Cartesian grid is considered, the equation can be simply rewritten in terms of fluxes at each numerical interface (between two cells):

$$
\frac{\partial f_{i, j}}{\partial t}+\frac{1}{\Delta x}\left(F_{i+\frac{1}{2}, j}-F_{i-\frac{1}{2}, j}+F_{i, j+\frac{1}{2}}-F_{i, j-\frac{1}{2}}\right)=\frac{1}{\tau}\left(M_{f_{i, j}}-f_{i, j}\right)
$$


with $F_{i+\frac{1}{2}, j}$ the numerical flux between cell $\Omega_{i, j}$ and cell $\Omega_{i+1, j}$ (with a similar notation for the other fluxes) which is expressed as :

$$
F_{i+\frac{1}{2}, j}=\max \left(0, \xi_{u}\right) f_{l}+\min \left(0, \xi_{u}\right) f_{r}
$$

with $f_{r}$ and $f_{l}$ the values of $f$ on the two sides of the interface and $\xi_{u}$ the first component of the microscopic velocity. The numerical expression of the distribution functions $f_{l}, f_{r}$ depends on the reconstruction used at the numerical interface. For a first order reconstruction, $f_{l}=f_{i, j}$ and $f_{r}=f_{i+1, j}$. For second order accuracy, a MUSCL reconstruction with slope limiters (MinMod for example) is employed:

$$
\left\{\begin{aligned}
f_{l} & =f_{i, j}+\frac{1}{2} \operatorname{MinMod}\left(f_{i+1, j}-f_{i, j}, f_{i, j}-f_{i-1, j}\right) \\
f_{r} & =f_{i+1, j}-\frac{1}{2} \operatorname{MinMod}\left(f_{i+1, j}-f_{i, j}, f_{i+2, j}-f_{i+1, j}\right)
\end{aligned}\right.
$$

\subsection{The time discretization}

The time discretization can be performed for all terms explicitly. But in this case, the time step will be determined by the space discretization $(\Delta x)$, the maximum velocity of the velocity grid and the relaxation time $\tau$. For small Knudsen numbers, the relaxation part becomes very stiff ( $\tau$ very small) and imposes a very strong restriction on the time step. Asher et al. [2] first presented IMEX schemes to cure this issue. Here, the IMEX scheme [28], [33] is chosen. The relaxation term is treated implicitly while the convective part is non stiff but highly non linear which means that an explicit scheme is more efficient.

The time integration for a $\nu$-stages IMEX Runge-Kutta scheme reads as follows:

$$
\begin{aligned}
f_{i, j}^{n+1} & =f_{i, j}^{n}-\Delta t \sum_{k=1}^{\nu} \tilde{\omega}_{k} \boldsymbol{\xi} \nabla_{\mathbf{x}} f_{i, j}^{(k)}+\frac{\Delta t}{\tau} \sum_{k=1}^{\nu} \omega_{k}\left(M_{f_{i, j}}^{(k)}-f_{i, j}^{(k)}\right) \\
f_{i, j}^{(k)} & =f_{i, j}^{n}-\Delta t \sum_{l=1}^{k-1} \tilde{A}_{k, l} \boldsymbol{\xi} \nabla_{\mathbf{x}} f_{i, j}^{(l)}+\frac{\Delta t}{\tau} \sum_{l=1}^{k} A_{k, l}\left(M_{f_{i, j}}^{(l)}-f_{i, j}^{(l)}\right) \\
f_{i, j}^{(1)} & =f_{i, j}^{n}+\frac{\Delta t}{\tau} A_{1,1}\left(M_{f_{i, j}}^{(1)}-f_{i, j}^{(1)}\right)
\end{aligned}
$$

where $A$ and $\tilde{A}$ are $\nu \times \nu$ matrices, with $\tilde{A}_{i, s}=0$ if $s \geq i$ and $A_{i, s}=0$ if $s>i$. These coefficients are derived from a double Butcher's tableaux:
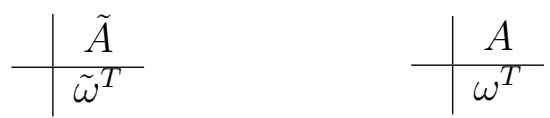

All the quantities until stage $k-1$ are known so the equation for stage $k$ becomes:

$$
\begin{aligned}
f_{i, j}^{(k)}= & \frac{\tau}{A_{k, k} \Delta t+\tau}\left(f_{i, j}^{n}-\Delta t \sum_{l=1}^{k-1} \tilde{A}_{k, l} \boldsymbol{\xi} \nabla_{\mathbf{x}} f_{i, j}^{(l)}+\frac{\Delta t}{\tau} \sum_{l=1}^{k-1} A_{k, l}\left(M_{f_{i, j}}^{(l)}-f_{i, j}^{(l)}\right)+\right. \\
& \left.\frac{A_{k, k} \Delta t}{\tau} M_{f_{i, j}}^{(k)}\right)
\end{aligned}
$$

where $f_{i, j}^{(k)}$ can be then computed explicitly since all the right hand side is known.In fact the Maxwellian at stage $k, M_{f_{i, j}}^{(k)}$ can be computed using the macroscopic variables at the previous 
stages, see [36].

We are interested in first and second order schemes, hence we use the IMEX schemes given by the following tableaux:

First-order scheme:
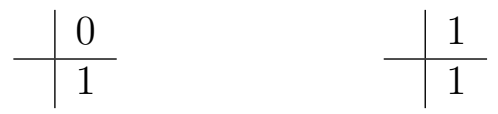

Second-order scheme:

\begin{tabular}{c|ccc}
0 & 0 & 0 \\
0 & 0 & 0 \\
0 & 1 & 0 \\
\hline 0 & $\frac{1}{2}$ & $\frac{1}{2}$
\end{tabular}

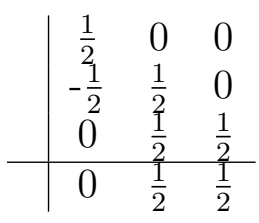

\section{The level set function}

When an immersed solid is considered on a Cartesian grid, one needs to apply the wall boundary condition on a surface that is arbitrarily crossing the grid. The discretization scheme we employ, directly uses geometric information delivered by the distance function defined on the Cartesian grid to reconstruct the solution near the solid boundaries.

To this end, the domain is decomposed in a fluid part and a solid part. In the solid the values of the physical variables are imposed in each cell since there is no calculation to perform. Such cells are called penalized cells. To decide whether or not a cell is penalized on a Cartesian mesh and to improve accuracy at the boundaries, we use the signed distance between a grid point and the immersed body. This distance is given by a specific level set function. Introduced by Osher and Sethian [32], the level set function implicitly defines the solid interface $\Sigma$ in the computational domain by its zero isoline. It is defined by:

$$
\phi(\mathbf{x})=\left\{\begin{aligned}
\operatorname{dist}_{\Sigma}(\mathbf{x}) & \text { outside the solid } \\
-\operatorname{dist}_{\Sigma}(\mathbf{x}) & \text { inside the solid }
\end{aligned}\right.
$$

where $\operatorname{dist}_{\Sigma}(\mathbf{x})$ is the minimum distance between the point considered (with coordinates $\mathbf{x}$ ) and the solid interface $\Sigma$.

Thanks to this function it is possible to compute the unit normal of the distance isoline through $x$, pointing towards the fluid as

$$
\mathbf{n}(\mathbf{x})=\frac{\nabla \phi(\mathbf{x})}{|\nabla \phi(\mathbf{x})|}
$$

For $\phi=0$, we have the unit normal to the interface, $\mathbf{n}_{w}(\mathbf{x})$.

In case of moving geometries, the level set function is convected with the imposed boundary velocity $\mathbf{u}_{\phi}$ :

$$
\partial_{t} \phi+\mathbf{u}_{\phi} \cdot \nabla \phi=0
$$

For the numerical test cases, when needed, this equation is solved with a WENO5 [23] discretization scheme in space and a standard Runge-Kutta 4 scheme for the integration in time.

Integrating (17) in time does not preserve the distance property of $\phi$. Therefore, a reinitialisation step is performed after each time integration step starting from the boundary $(\phi=0)$. In our case, this is done via a Fast Marching algorithm [38]. 


\section{$5 \quad$ Wall boundary conditions}

Two kinds of boundary conditions for kinetic models are usually found in the literature: the diffuse boundary condition and the specular reflection.

The diffuse boundary condition model assumes that the solid is in equilibrium with the fluid in contact with the wall. The distribution function for the fluid is therefore described by a wall Maxwellian distribution function, $M_{w}$, computed with given temperature and velocity of the wall.

The specular reflection models a wall at which particles are merely reflected. There is no mass and energy fluxes through the wall (impermeability condition). The distribution function $f_{s}$, corresponding to this boundary condition is a reflection of the distribution function coming from the fluid.

Both models can be taken into account using an accommodation coefficient $\alpha \in[0,1]$ to create the boundary (Maxwell, [8]) model:

$$
f_{b}=\alpha M_{w}+(1-\alpha) f_{s}
$$

where $f_{b}$ is the distribution function representing the actual wall model. Next, we consider the discretization of these boundary conditions.

The coefficient $\alpha$ depends on the gas and the solid considered, through the gas-surface interaction model chosen. A review on gas-surface interactions can be found in [20]. In this work we focus on the asymptotic behaviour of the scheme towards Euler equations with particular emphasis on the asymptotic behaviour of the boundary condition. Imposing a temperature and a velocity at the wall cannot provide an asymptotic preserving boundary condition, because in Euler equations only the component of the velocity normal to the wall can be prescribed. Hence, the case $\alpha>0$ cannot provide an asymptotic preserving boundary condition. Thus, to make sure that the boundary condition preserves the Euler limit, it is necessary that $\alpha \rightarrow 0$ close to the hydrodynamic regime. However, as our results will show, this is not enough to obtain an AP boundary condition. In the following we will show how to modify specular reflection to preserve the Euler limit. For this reason, in the numerical tests, we will concentrate on the case $\alpha=0$. However, for completeness, we also present the diffuse boundary condition to include the case of non equilibrium flows, far from the Euler limit.

\subsection{The diffuse boundary condition}

We use this wall model in the formulation of the Euler-AP boundary condition, see section 5.2.2. The diffuse condition is imposed through a Maxwellian distribution function. Boundary conditions prescribe temperature, velocity and zero mass flux. The wall Maxwellian is first computed with a density of 1 , temperature and velocity of the wall. Then by invoking mass conservation through the wall, one can recover the density $\rho_{w}$. Indeed, mass conservation near the wall can be written

$$
F_{\text {in }}+F_{\text {out }}=0
$$

with $\mathrm{F}$ the mass flux with subscript "in" to denote the flux going towards the wall and "out" the flux going towards the fluid at the physical interface. Then $F_{\text {out }}$ corresponds to the quantity of mass going towards the fluid with the wall characteristics (density, temperature, velocity) and with a Maxwellian distribution $M_{w}$, called the wall Maxwellian. We have:

$$
\int_{\left(\boldsymbol{\xi}-\mathbf{U}_{w}\right) \cdot \mathbf{n}_{w}<0}\left(\boldsymbol{\xi}-\mathbf{U}_{w}\right) \cdot \mathbf{n}_{w} f_{w} d \boldsymbol{\xi}+\int_{\left(\boldsymbol{\xi}-\mathbf{U}_{w}\right) \cdot \mathbf{n}_{w}>0}\left(\boldsymbol{\xi}-\mathbf{U}_{w}\right) \cdot \mathbf{n}_{w} M_{w} d \boldsymbol{\xi}=0
$$


with $f_{w}$ the distribution function near the wall (for example the one in the closest fluid cell), $M_{w}$ the wall Maxwellian and $\mathbf{n}_{w}$ the normal to the wall pointing towards the fluid. This equation can also be expressed in terms of the wall macroscopic quantities (velocity $\mathbf{U}_{w}$, temperature $T_{w}$ and density near the wall $\rho_{w}$ ):

$$
\begin{gathered}
\int_{\mathbf{c}_{w} \cdot \mathbf{n}_{w}<0} \mathbf{c}_{w} \cdot \mathbf{n}_{w} f_{w} d \boldsymbol{\xi}+\rho_{w} \int_{\mathbf{c}_{w} \cdot \mathbf{n}_{w}>0} \frac{\mathbf{c}_{w} \cdot \mathbf{n}_{w}}{\left(2 \pi T_{w}\right)^{3 / 2}} \exp \left(-\frac{\left|\boldsymbol{\xi}-\mathbf{U}_{w}\right|^{2}}{2 T_{w}}\right) d \boldsymbol{\xi}=0 \\
\int_{\mathbf{c}_{w} \cdot \mathbf{n}_{w}<0} \mathbf{c}_{w} \cdot \mathbf{n}_{w} f d \boldsymbol{\xi}+\rho_{w} \int_{\mathbf{c}_{w} \cdot \mathbf{n}_{w}>0} \mathbf{c}_{w} \cdot \mathbf{n}_{w} \overline{M_{w}} d \boldsymbol{\xi}=0
\end{gathered}
$$

with $\mathbf{c}_{w}=\boldsymbol{\xi}-\mathbf{U}_{w}$ and $\overline{M_{w}}$ the wall Maxwellian corresponding to a density of 1 . Then, $\overline{M_{w}}$ can be computed with a Newton-Raphson algorithm (discrete Maxwellian).

Then the density $\rho_{w}$ can be calculated as :

$$
\rho_{w}=-\frac{\int_{\left(\boldsymbol{\xi}-\mathbf{U}_{w}\right) \cdot \mathbf{n}_{w}<0}\left(\boldsymbol{\xi}-\mathbf{U}_{w}\right) \cdot \mathbf{n}_{w} f d \boldsymbol{\xi}}{\int_{\left(\boldsymbol{\xi}-\mathbf{U}_{w}\right) \cdot \mathbf{n}_{w}>0}\left(\boldsymbol{\xi}-\mathbf{U}_{w}\right) \cdot \mathbf{n}_{w} \overline{M_{w}} d \boldsymbol{\xi}}
$$

and

$$
M_{w}=\rho_{w} \overline{M_{w}}
$$

\subsection{Impermeability boundary condition}

Here the impermeability condition is considered in the sense of Euler equations. Through the boundary there is no mass flux and no energy flux. We first describe how this boundary condition is typically imposed by specular reflection of the distribution function. Then a novel Euler-AP condition method will be introduced for the inviscid limit.

\subsubsection{Specular reflection}

Each particle hitting the wall is immediately reflected by the wall with the same tangential velocity and the opposite normal velocity :

$$
\boldsymbol{\xi}_{\text {refl }}=\boldsymbol{\xi}-2\left(\left(\boldsymbol{\xi}-\mathbf{U}_{w}\right) \cdot \mathbf{n}_{w}\right) \mathbf{n}_{w}
$$

with $\boldsymbol{\xi}_{\text {refl }}$ the particle velocity after reflection, $\boldsymbol{\xi}$ the particle velocity before reflection, $\mathbf{U}_{w}$ the wall velocity and $\mathbf{n}_{w}$ the normal to the wall. This holds true for each particle such that $\boldsymbol{\xi} \cdot \mathbf{n}_{w}>0$ (from now, $\mathbf{U}_{w}$ is set to zero for simplicity). For $\boldsymbol{\xi} \cdot \mathbf{n}_{w}<0$, the distribution function on the boundary is already known and equal to the one in the fluid cell. The distribution function for the boundary condition has to be computed only for $\boldsymbol{\xi} \cdot \mathbf{n}_{w}>0$. The entire distribution function $f_{s}$ enforcing the boundary condition is then:

$$
f_{s}=\left\{\begin{array}{l}
f \text { for } \quad \boldsymbol{\xi} \cdot \mathbf{n}_{w}<0 \\
f\left(\boldsymbol{\xi}_{\text {refl }}\right) \text { for } \quad \boldsymbol{\xi} \cdot \mathbf{n}_{w}>0
\end{array}\right.
$$

In the reduced model, the same procedure is applied to $\phi$ and $\psi$. This guarantees zero mass and energy fluxes:

$$
\begin{aligned}
F_{\text {mass }} & =\int_{\boldsymbol{\xi} \cdot \mathbf{n}_{w}<0} \boldsymbol{\xi} \cdot \mathbf{n}_{w} f(\boldsymbol{\xi}) d \boldsymbol{\xi}+\int_{\boldsymbol{\xi} \cdot \mathbf{n}_{w}>0} \boldsymbol{\xi} \cdot \mathbf{n}_{w} f\left(\boldsymbol{\xi}_{\text {refl }}\right) d \boldsymbol{\xi} \\
& =\int_{\boldsymbol{\xi} \cdot \mathbf{n}_{w}<0} \boldsymbol{\xi} \cdot \mathbf{n}_{w} f(\boldsymbol{\xi}) d \boldsymbol{\xi}+\int_{\boldsymbol{\xi} \cdot \mathbf{n}_{w}<0}-\boldsymbol{\xi} \cdot \mathbf{n}_{w} f(\boldsymbol{\xi}) d \boldsymbol{\xi} \\
& =0
\end{aligned}
$$




$$
\begin{aligned}
F_{\text {energy }} & =\int_{\boldsymbol{\xi} \cdot \mathbf{n}_{w}<0}|\boldsymbol{\xi}|^{2} \boldsymbol{\xi} \cdot \mathbf{n}_{w} f(\boldsymbol{\xi}) d \boldsymbol{\xi}+\int_{\boldsymbol{\xi} \cdot \mathbf{n}_{w}>0}|\boldsymbol{\xi}|^{2} \boldsymbol{\xi} \cdot \mathbf{n}_{w} f\left(\boldsymbol{\xi}_{\text {refl }}\right) d \boldsymbol{\xi} \\
& =\int_{\boldsymbol{\xi} \cdot \mathbf{n}_{w}<0}|\boldsymbol{\xi}|^{2} \boldsymbol{\xi} \cdot \mathbf{n}_{w} f(\boldsymbol{\xi}) d \boldsymbol{\xi}+\int_{\boldsymbol{\xi} \cdot \mathbf{n}_{w}<0}-|\boldsymbol{\xi}|^{2} \boldsymbol{\xi} \cdot \mathbf{n}_{w} f(\boldsymbol{\xi}) d \boldsymbol{\xi} \\
& =0
\end{aligned}
$$

However, because of the discretization of the velocity space, one needs to compute $f\left(\boldsymbol{\xi}_{\text {refl }}\right)$ where in general $\boldsymbol{\xi}_{\text {refl }}$ does not correspond to a collocation point. In other words, even though $\boldsymbol{\xi}$ is a grid point, $\boldsymbol{\xi}_{\text {refl }}$ is not a velocity grid point except if the wall is parallel to the grid. Therefore $\boldsymbol{\xi}_{\text {refl }}$ must be interpolated. The numerical experiments show that the interpolation must be higher-order accurate to guarantee zero mass and energy fluxes at the wall to an acceptable degree of approximation.

In the $1 \mathrm{D}$ case, this problem does not arise. It is enough to have a velocity grid symmetric with respect to $\xi_{u}=0$. Specular reflection reduces then to choose $f_{s}\left(\xi_{u}\right)=f\left(-\xi_{u}\right)$ in order to build the reflected distribution function.

In $2 \mathrm{D}$, due to interpolation errors, mass and energy fluxes are not identically zero at the walls. This error leads to a boundary layer in the limit of inviscid gas simulations that should not exist. As shown in the numerical illustrations, to remove this spurious effect it is possible to use either a finer velocity grid or a higher-order interpolation, at the price of significantly larger computational costs.

\subsubsection{A new discrete Euler-AP impermeability condition}

Let us assume that the distribution function is a Maxwellian ( $K n$ number close to 0 ). Then, imposing the impermeability condition at the wall corresponds to impose a Maxwellian distribution function at the wall exactly as described in section 5.1. However, in this case the Maxwellian distribution must be built with macroscopic quantities computed from the fluid, thus the velocity must have the same tangential component of the fluid next to the boundary with zero wall-normal component and the temperature must be the same of the fluid. Therefore, in contrast with what is done in section 5.1, tangential velocity and temperature are extrapolated from the fluid to the wall. Let $f_{M}$ be the Maxwellian distribution function at the wall computed using the fluid properties.

Eventually, the diluted fluid next to the wall can be considered Maxwellian only in the limit of the continuum regime. To build a fully asymptotic preserving boundary condition valid in more rarefied regimes, this model is included in equation (18) with a new coefficient $\beta$ :

$$
f_{b}=\alpha M_{w}+(1-\alpha)\left(\beta f_{s}+(1-\beta) f_{M}\right)
$$

with $\beta \in[0,1]$ and such that it is close to zero in the inviscid limit for $K n \rightarrow 0$ and $f_{M}$ is the distribution function built along the lines described above. If $K n$ is not close to zero, the classic specular reflection $\left(\beta \rightarrow 1\right.$ and $\left.f_{b} \rightarrow \alpha M_{w}+(1-\alpha) f_{s}\right)$ correctly takes over. However we recall that to have an asymptotic preserving boundary condition, $\alpha$ should be set to zero. In (21), the fully asymptotic preserving boundary condition is presented in a more general framework in which, for example, $\alpha$ depends on the Knudsen number such that $\alpha$ goes to 0 as $K n$ goes to 0 .

To set the value of $\beta$ we emphasize that $f_{M}$ corresponds to the specular reflection only when the distribution function in the fluid is close to a Maxwellian. If it is not the case, the specular reflection is computed with $f_{s}$ (in particular in the rarefied regime). Thus, in our model, $\beta$ is set as follows:

$$
\beta=\min \left(1, \frac{\left\|f-M_{f}\right\|_{L^{2}}}{\max (f) t o l}\right)
$$


with $t o l$ a tolerance on the distance in $L^{2}$ norm between the distribution function in the closest cell immersed in the fluid $f$ and its corresponding Maxwellian. Thus, if $\frac{\left\|f-M_{f}\right\|_{L^{2}}}{\max (f)}<t o l$, the specular reflection fully corresponds to the Euler-AP boundary condition. Conversely, in the rarefied regime, $f$ is far from the Maxwellian, then $\beta=1$ and the classical specular reflection correctly takes over. The tolerance tol is used to reduce the sensitivity of the algorithm to $\left\|f-M_{f}\right\|$. To ensure the correct asymptotic behaviour, when $f$ is sufficiently close to the Maxwellian, $\beta$ must tend to 0 . The value of tol does not need to be precisely set since if $f$ is far from the Maxwellian, numerical errors are introduced by both the classical specular reflection (due to interpolations) and the new Euler-AP condition since it is not exactly the reflection of $f$. Thus, a criterion to correctly set tol, is that the two sources of error should balance, that is tol $\sim \Delta \xi_{u}^{2}$ which is the order of the interpolation error, if the trapezoid rule is used for quadrature.

\subsection{Wall boundary condition on Cartesian grid}

In this section we describe the scheme to impose the Euler-AP boundary condition of section 5.2.2 to immersed boundaries on Cartesian grids. The diffuse and the specular reflection schemes are similar. Also, it should be noted that this technique can be easily applied to the case of body fitted grids.

In the case of a solid immersed in the flow, a fictitious state has to be created in the solid to compute the transport step numerically between a fluid cell and a cell containing the solid. The idea is first to compute the equivalent distribution function at the solid interface satisfying the imposed boundary condition and then create a fictitious state in the neighbour solid cell called ghost cell that respects the boundary value at a given approximation order. To do so, a few parameters on the boundary are needed. These parameters are presented in figure 1.

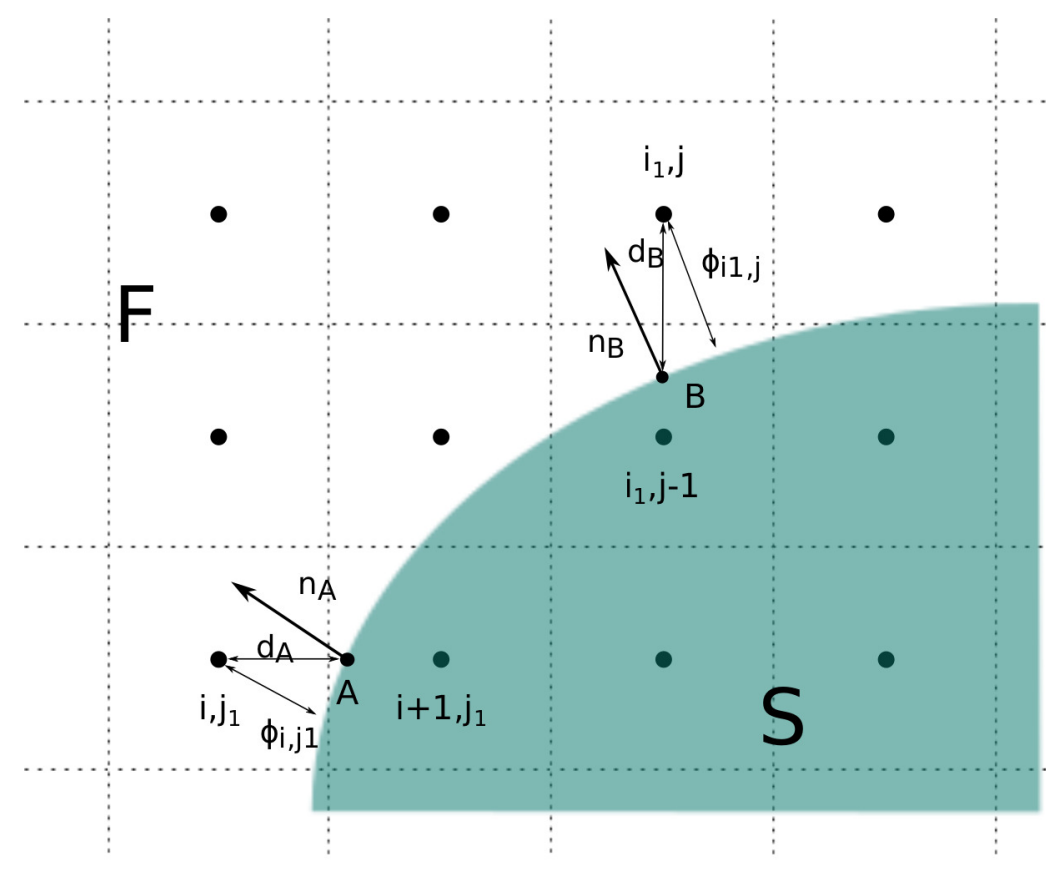

Figure 1: Immersed interface on a Cartesian mesh.

In each grid point, the shortest distance to the boundary $\phi$ is known through the levelset 
function (eq.15). One can also compute the normal $\mathbf{n}$ to the boundary in each grid point thanks to (16). In the following, we distinguish the interface between two cells and the interface between the fluid and the solid. The first interface will be called numerical interface (where the numerical fluxes are computed) while the latter will be called the physical interface (where the boundary condition is imposed).

The problem reduces to determine a Maxwellian distribution function on the boundary and extrapolate or interpolate it to the numerical interface. The method will be described for the new impermeability condition but the second part of the method (transport at the numerical interface) can be easily transposed for any condition where the distribution function is known at the boundary (specular reflection, diffuse condition).

The point A will be used to compute the interpolation at the interface $i+1 / 2, j 1$. The fluxes at interface $i 1, j-1 / 2$ will be computed by calculating the wall Maxwellian on point B. Since the same scheme is used on points $\mathrm{A}$ and $\mathrm{B}$, the method will be explained on point $\mathrm{A}$.

\subsubsection{First order Euler-AP scheme}

To compute the fictitious state at first order, the Maxwellian distribution function at the wall is built as presented previously in 5.1 with the tangential velocity $\mathbf{U}_{A} \cdot \boldsymbol{\tau}_{i, j 1}$ and temperature $T_{A}$ taken from the fluid cell and a zero relative normal velocity $\left(\mathbf{U}_{A}-\mathbf{U}_{w}\right) \cdot \mathbf{n}_{i, j 1}$ :

$$
\left\{\begin{array}{l}
T_{A}=T_{i, j 1} \\
\left(\mathbf{U}_{A}-\mathbf{U}_{w}\right) \cdot \boldsymbol{\tau}_{i, j 1}=\left(\mathbf{U}_{i, j 1}-\mathbf{U}_{w}\right) \cdot \boldsymbol{\tau}_{i, j 1} \\
\left(\mathbf{U}_{A}-\mathbf{U}_{w}\right) \cdot \mathbf{n}_{i, j 1}=0
\end{array}\right.
$$

The density $\rho_{A}$ is calculated thanks to the distribution function in cell $(i, j 1)$ invoking mass conservation through the wall as in (19). The Maxwellian built with $\rho_{A}, \mathbf{U}_{A}, T_{A}$ is then simply imposed as the state in the first solid cell $(i+1, j 1)$.

\subsubsection{Second order Euler-AP scheme}

The main idea is now to impose the boundary condition on the physical interface and reconstruct the conditions at the numerical interface with second-order accuracy.

The impermeability boundary condition is applied at the physical interface by imposing a Maxwellian distribution function. This distribution function depends on the temperature, the velocity and the distribution function in the fluid. All these information are extrapolated from the fluid. To find the position of the wall, the levelset function is used and the distance $d_{A}$ between the cell center and the wall is known as:

$$
d_{A}=\frac{\Delta x\left|\phi_{i, j 1}\right|}{\left|\phi_{i, j 1}\right|+\left|\phi_{i+1, j 1}\right|}
$$

where $\phi_{i, j 1}$ (respectively $\left.\phi_{i+1, j 1}\right)$ is the distance between the point $(i, j 1)$ (respectively $(i+1, j 1)$ ) and the boundary and $\Delta x$ is the space grid step. The normal can also be computed by

$$
\mathbf{n}_{A}=\mathbf{n}_{i, j 1}+\frac{d_{A}}{\Delta x}\left(\mathbf{n}_{i+1, j 1}-\mathbf{n}_{i, j 1}\right)
$$

where $\mathbf{n}_{i, j 1}$ (respectively $\mathbf{n}_{i+1, j 1}$ ) is the normal to the boundary in point $(i, j 1)$ (respectively $(i+1, j 1))$.

The temperature and the velocity now can be extrapolated with a second order polynomial using $T_{i, j 1}, T_{i-1, j 1}, T_{i-2, j 1}$ and $\mathbf{U}_{i, j 1}, \mathbf{U}_{i-1, j 1}, \mathbf{U}_{i-2, j 1}$. The wall temperature $T_{e x t}$ is the result 
of the extrapolation while the wall velocity $\mathbf{U}_{\text {ext }}$ is only equal to the tangential part of the extrapolated velocity such that the impermeability condition $\left(\left(\mathbf{U}_{A}-\mathbf{U}_{w}\right) \cdot \mathbf{n}_{A}=0\right)$ is enforced:

$$
\left\{\begin{array}{l}
T_{A}=T_{e x t} \\
\mathbf{U}_{A}=\mathbf{U}_{e x t}-\left(\left(\mathbf{U}_{e x t}-\mathbf{U}_{w}\right) \cdot \mathbf{n}_{A}\right) \mathbf{n}_{A}
\end{array}\right.
$$

To extrapolate the distribution function at the boundary in order to compute the density, an upwind reconstruction is used. For each microscopic velocity $\boldsymbol{\xi}$ such that $\boldsymbol{\xi} \cdot \mathbf{n}_{A}<0 f(\boldsymbol{\xi})$ is set as:

$$
f_{A}(\boldsymbol{\xi})=f_{n, m}(\boldsymbol{\xi}) \quad \text { such that } \frac{\mathbf{x}_{A}-\mathbf{x}_{n, m}}{\left\|\mathbf{x}_{A}-\mathbf{x}_{n, m}\right\|} \cdot \frac{\boldsymbol{\xi}}{\|\boldsymbol{\xi}\|}=\max _{(k, l) \in \Upsilon_{i, j 1}}\left(\frac{\mathbf{x}_{A}-\mathbf{x}_{k, l}}{\left\|\mathbf{x}_{A}-\mathbf{x}_{k, l}\right\|} \cdot \frac{\boldsymbol{\xi}}{\|\boldsymbol{\xi}\|}\right)
$$

with $\mathbf{x}_{A}$ the position of the boundary $\left(\mathbf{x}_{A}=\mathbf{x}_{i, j 1}-d_{A} \mathbf{n}_{A}\right)$ and $\Upsilon_{i, j 1}$ containing all the fluid neighbours of cell $(i, j 1)$. A graphic illustration is given in figure 2. In this example, the velocity grid has 8 grid points in $2 \mathrm{D}$. The third dimension in velocity is eliminated with the reduced model, which, we recall, is exact (see section 2.2). The distribution function is required in $\mathrm{A}$ for $\boldsymbol{\xi}_{1}, \boldsymbol{\xi}_{6}, \boldsymbol{\xi}_{7}, \boldsymbol{\xi}_{8}$. Here $\Upsilon_{i, j}$ is represented in blue. One can create $f_{A}$ for $\boldsymbol{\xi} \cdot \mathbf{n}_{A}<0$ as :

$$
\left\{\begin{aligned}
f_{A}\left(\boldsymbol{\xi}_{1}\right) & =f_{i, j}\left(\boldsymbol{\xi}_{1}\right) \\
f_{A}\left(\boldsymbol{\xi}_{6}\right) & =f_{i+1, j+1}\left(\boldsymbol{\xi}_{6}\right) \\
f_{A}\left(\boldsymbol{\xi}_{7}\right) & =f_{i+1, j+1}\left(\boldsymbol{\xi}_{7}\right) \\
f_{A}\left(\boldsymbol{\xi}_{8}\right) & =f_{i, j+1}\left(\boldsymbol{\xi}_{8}\right)
\end{aligned}\right.
$$

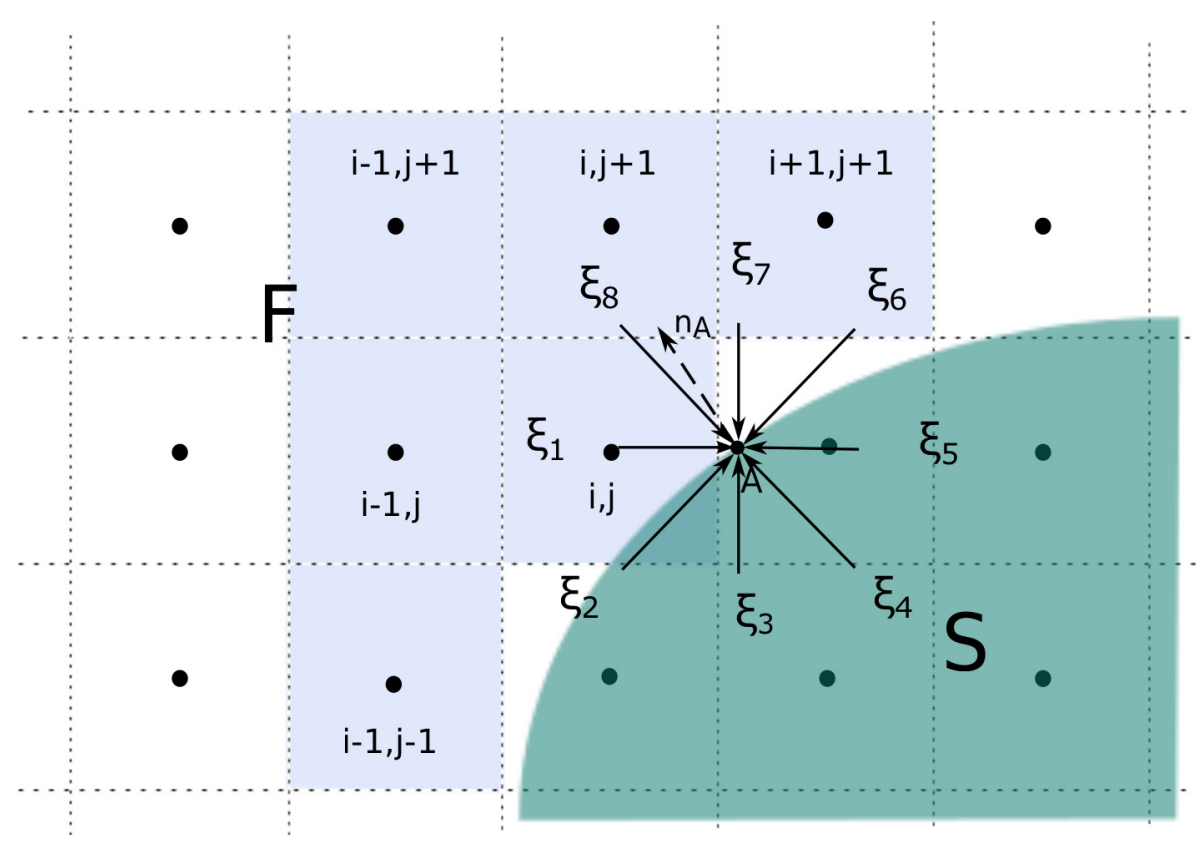

Figure 2: Graphic illustration of the reconstruction for a 2D 8 velocities grid.

It is worth to remark that this reconstruction avoids interpolation but is not formally secondorder accurate. A MUSCL-type reconstruction could be applied instead but an upwind reconstruction has the advantage of taking into account the main direction of the flux. Indeed, a reconstruction with slopes chosen as in (13) does not prevent the possibility of selecting a downwind reconstruction which might lead to high extrapolation errors. 
The part of $f$ for $\boldsymbol{\xi} \cdot \mathbf{n}_{A}>0$ corresponds to microscopic velocities coming from the wall and is not required for the computation of the wall Maxwellian $f_{M_{A}}$.

Finally, the distribution function used as boundary condition can be computed as follows:

$$
f_{b}=\left\{\begin{array}{l}
f_{A} \text { for } \boldsymbol{\xi} \cdot \mathbf{n}_{A}<0 \\
f_{M_{A}} \text { for } \boldsymbol{\xi} \cdot \mathbf{n}_{A}>0
\end{array}\right.
$$

Once the distribution for the boundary condition is known at the wall, it has to be interpolated or extrapolated to the numerical interface with a linear reconstruction to preserve second order. One can use the same slope as in the fluid cell and the exact distance between the numerical (between cells) and physical interface $\left(d_{A}\right.$ on figure 1$)$ :

$$
f_{i+1 / 2, j 1, r}=f_{b}+\frac{d_{A}}{\Delta x} \operatorname{MinMod}\left(f_{i, j 1}-f_{i-1, j 1}, f_{i-1, j 1}-f_{i-2, j 1}\right)
$$

The fluxes at the numerical interface can be now computed as usual.

\section{Numerical results}

The accuracy of the Euler-AP boundary condition is initially tested in 1D for cases where the reference solution is exact, section 6.1. In 2D, we consider well known test cases both on a body fitted mesh (section 6.2) and on a Cartesian mesh (section 6.3), in the continuum regime. Second-order accuracy of the method is illustrated on the Ringleb flow (section 6.4). Furthermore, we compare the Euler-AP wall model to numerical results in the literature in the rarefied regime (section 6.5). Finally, we simulate a nozzle plume with coexisting continuum and rarefied regime to qualitatively validate the Euler-AP boundary condition against experimental data.

Since we are mainly interested in close to equilibrium flows and in particular the asymptotic behaviour towards the hydrodynamic regime, we take $\alpha=0$ in (18). Moreover, the parameter tol is always tol $=10^{-2}$ according to the criteria described in section 5.2.2. If the velocity space step is such that $\Delta \xi_{u}^{2}$ is not of the order of $10^{-2}$ the tolerance has to be modified ensuring that $t o l \simeq \Delta \xi_{u}^{2}$. In order to focus on the spurious effects due to a treatment of the boundary condition which is not AP, and on the improvement we can obtain, in most cases the Knudsen numbers tested are very small.

\subsection{Reflection of a rarefaction wave and a shock wave}

Here, we will test the precision of the new Euler-AP method with $\alpha=0$ and $\beta=0$ for typical 1D problems for which the exact solution is known. In particular, we consider the reflection of a rarefaction wave and a shock wave on a solid wall for a monoatomic gas at equilibrium. The boundary condition applied at the wall is the impermeability condition with the Euler-AP method. One can note that the classical way to implement it (specular reflection) perfectly works here since there is no need to interpolate in the velocity space. For the rarefaction wave, the exact solution is computed using Riemann invariants with a velocity $v=-0.35$ assigned to the left of the rarefaction wave (see Figure 3, left). To the right, where the gas is steady, the pressure is 1 and the speed of sound is 1 . For the shock, the Rankine-Hugoniot conditions and an exact Riemann problem at the wall are used (see Figure 3, right). The shock wave propagates at Mach 1.2. $K n_{\infty}$ is set to $10^{-10}$, to ensure that the gas in in equilibrium, and we only see the error due to the boundary condition. 
The domain goes from 0 to 3 and the wave is generated at $t=0$ in 0 . The wall position is set to $x=3$. In order to compute the error of our scheme with respect to the analytical solution, the grid points (center of each cell) are shifted with respect to the wall position as follows:

$$
x_{i, j}=x_{i, j}+\psi \Delta x
$$

with $\Delta x$ the space step and $\psi$ a coefficient varying from 0 to 1 . When $\psi=0.5$, the position of the wall exactly corresponds to a numerical interface. In this case, the error with respect to the analytical solution is optimal.

Numerical simulations are performed with 100 space grid points and 50 grid points in velocity, with an initial data $\left(t_{s}=0\right)$ computed analytically (for Euler equations) at $\mathrm{t}=1.8$ for the rarefaction wave and $\mathrm{t}=1$ for the shock wave. The simulation is stopped at $t_{s}=3$ and $t_{s}=2$ respectively, when the wave is completely reflected by the wall. This is equivalent to the analytical solution at $t=4.8$ for the rarefaction wave and $t=3$ for the shock wave.
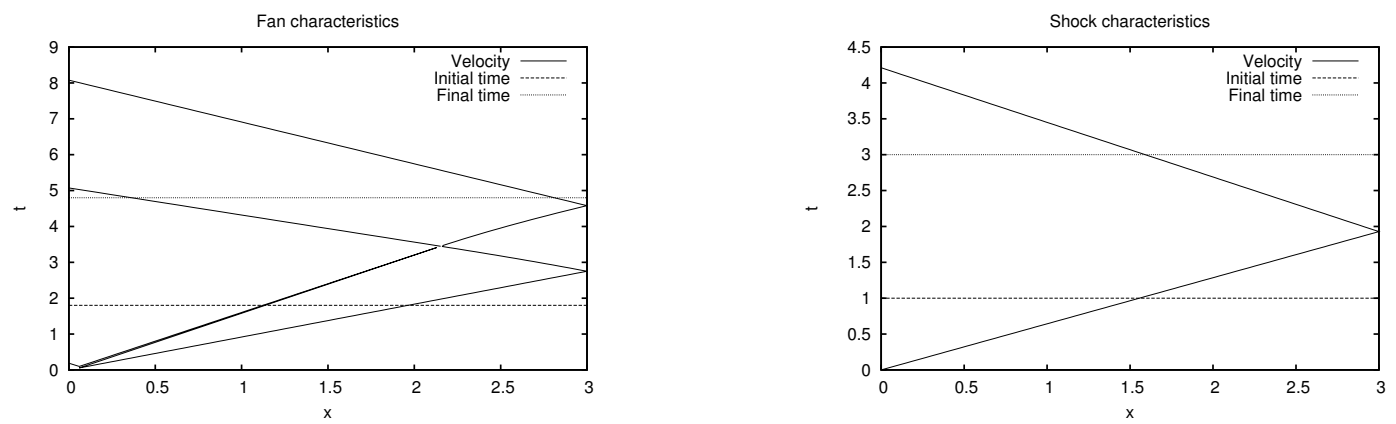

Figure 3: Characteristics for the reflection of a rarefaction wave (left) and a shock wave (right).

Errors in $L_{1}, L_{2}$ and $L_{\infty}$-norm for the velocity as a function of $\psi$ (the position of the wall), have been computed and shown on Figure 4.
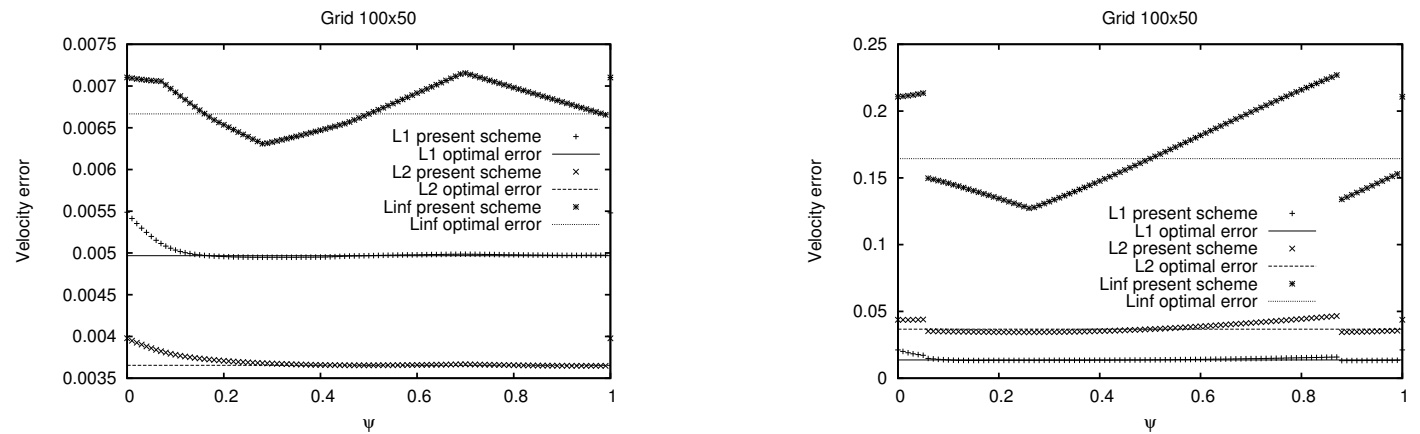

Figure 4: Velocity error for the reflection of a rarefaction wave (left) and a shock wave (right).

We observe that the errors in $L_{1}$ and $L_{2}$ norms increase as $\psi$ goes to 0 because the macroscopic quantities are extrapolated over larger distances. However, they remain close to the optimal error. For the three norms, the error for $\psi=0$ and $\psi=1$ is the same because it represents the same configuration.

\subsection{The oblique shock}

Here, we will first compare the specular reflection described in sect.5.2.1 with the new EulerAP method with $\beta=0$ in (21) (and still $\alpha=0$ ) on a 2D exact test case, using a body-fitted 
grid. Hence, we only compare the two ways of imposing specular reflection (the classical way with interpolation and the Maxwellian distribution function). Then a sensibility analysis with respect to the parameter tol is carried out. A wedge reflect an incoming horizontal flux at $M=2.324$. The grid is body fitted to deal with the single issue of the boundary condition without the influence of the Cartesian grid scheme. The angle of the wall is 10 degrees. The initial condition is $u=3, v=0, p=1, T=1$ in dimensionless form and $K n_{\infty}=10^{-8}$. The same condition is imposed at inlet and the impermeability condition is applied on the body. A shock is created on the body and moves in the fluid. The analytical solution for the angle of the shock $(\delta)$, Mach number, density, pressure and temperature after the shock is known:

$$
\left\{\begin{array}{l}
\delta=35.2326 \\
M=1.818 \\
\rho=1.4987 \\
P=1.9969 \\
T=1.3324
\end{array}\right.
$$

Two specular reflections schemes have been tested with different interpolation methods and compared to the new Euler-AP method. The first one uses a bilinear interpolation. The second one uses a bicubic interpolation. Results are shown for a grid $100 \times 100$ in space and $21 \times 21$ in velocity space (and $81 \times 81$ for the bilinear interpolation).

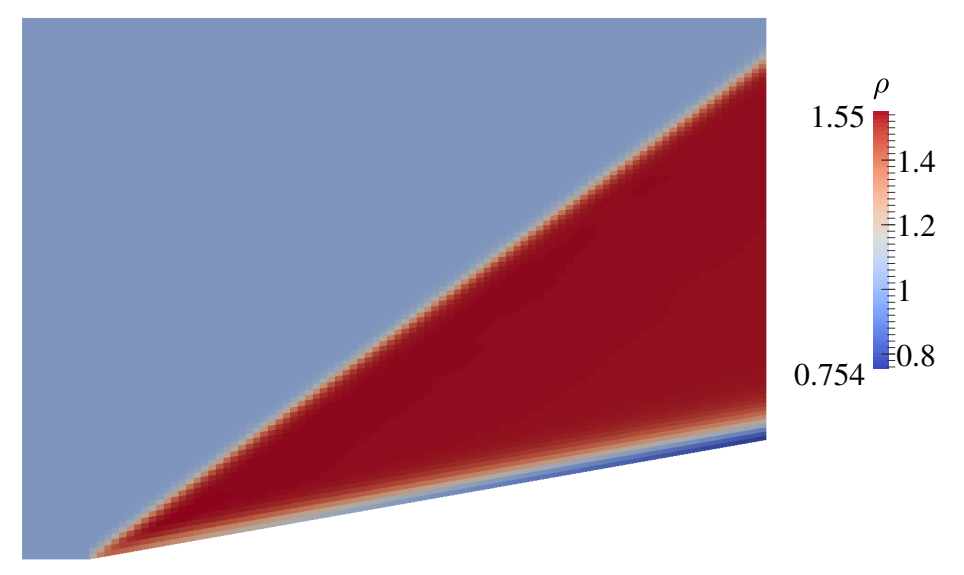

Figure 5: Density solution for the oblique shock with bilinear interpolation on $21 \times 21$ grid points.

The layer developing close to the wall with the bilinear interpolation (see figure 5) is due to a spurious energy flux across the wall (100 times larger than for the present method, see figure 8). This energy flux is caused by interpolation errors. In fact, when the velocity grid is refined, the spurious layer tends to decrease as we can see using $81 \times 81$ points on the velocity grid (see figure 6).

The layer is much smaller in the case of a bicubic interpolation (see figure 6.2) because the spurious energy flux across the wall is much lower and gives results in better accordance with the theory. Again, it shows that this spurious layer is only a numerical artefact.

The Euler-AP method does not show this kind of layer on the boundary (see figure 8 ). The solution is uniform after the shock.

On a horizontal line $(y=0.3)$, one can compare the different results with respect to the analytical solution. Figure 9 shows the comparison of the solutions for the pressure and the 


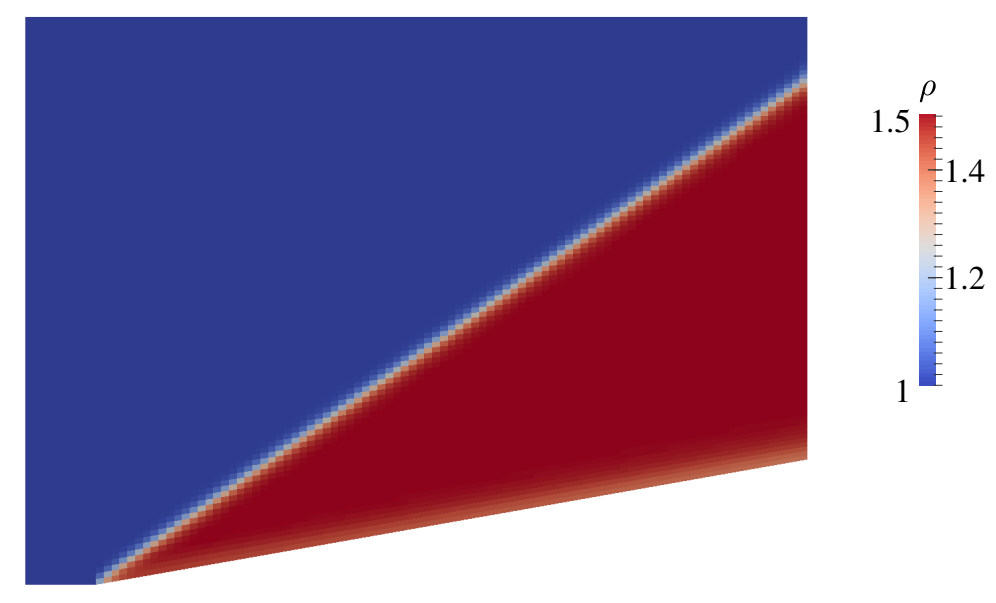

Figure 6: Density solution for the oblique shock with bilinear interpolation on $81 \times 81$ grid points.

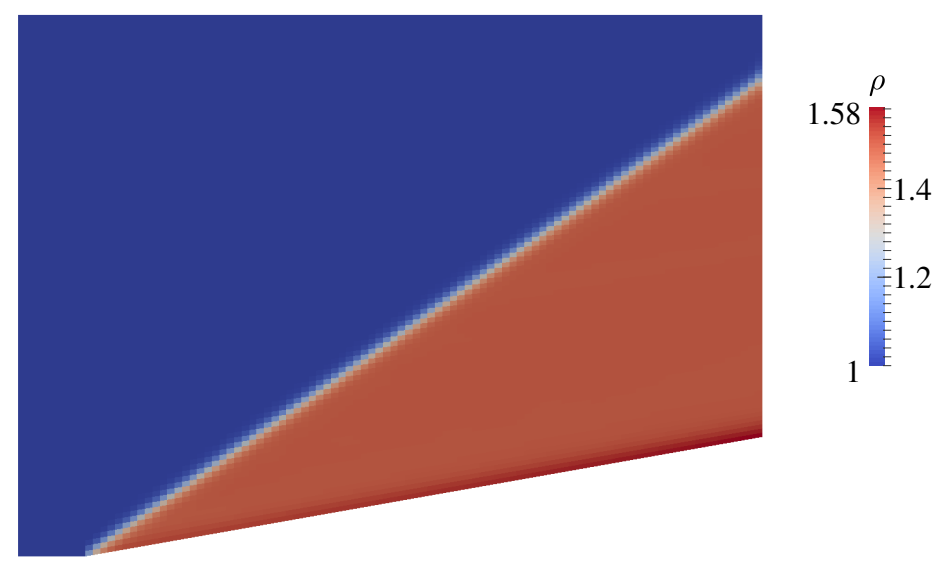

Figure 7: Density solution for the oblique shock with a bicubic interpolation with $21 \times 21$ velocity grid points.

temperature given by each method with $21 \times 21$ points in velocity. The exact solution (for Euler equations) is represented with a solid line.

For all methods, the position of the shock is correct but the values for the pressure and the temperature after the shock are different. The Euler-AP method and the specular reflection with a bicubic interpolation are clearly more accurate. Zooming on these pictures (see figure 10) we can see that the results obtained by the Euler-AP method are even more accurate than the ones obtained with the bicubic interpolation.

Note that these spurious overestimated post-shock values arise from the error in the boundary condition and propagate in the bulk of the fluid affecting the whole solution.

On the same test case, we also tested the sensibility of the solution with respect to the value of tol. We now impose the boundary condition still through formula (21) but with $\beta$ set as in (22). We recall that to get the correct asymptotic behaviour towards the hydrodynamic regime, we still impose $\alpha=0$. The error on the temperature in $L_{1}$ norm is shown on figure 11 . It 


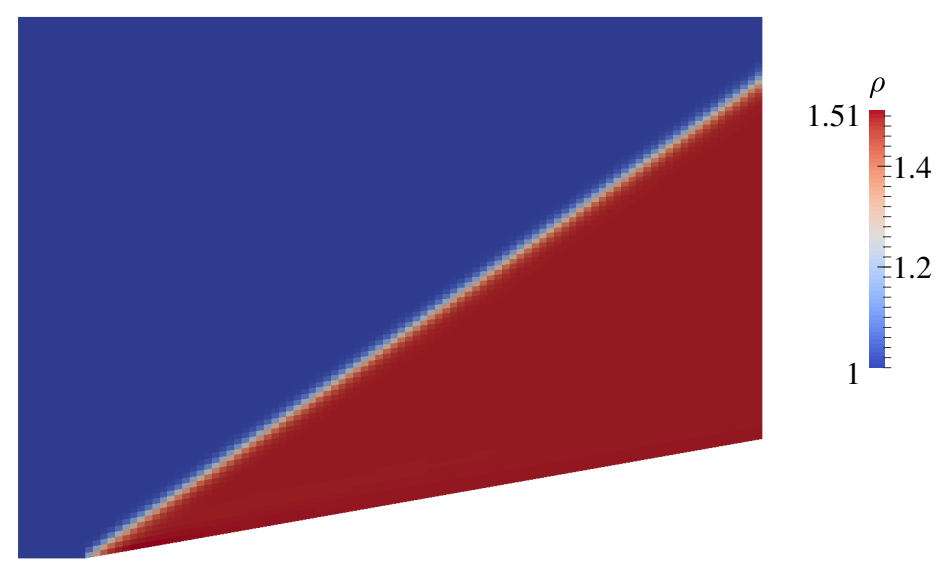

Figure 8: Density for the oblique shock with the present method $(21 \times 21)$.
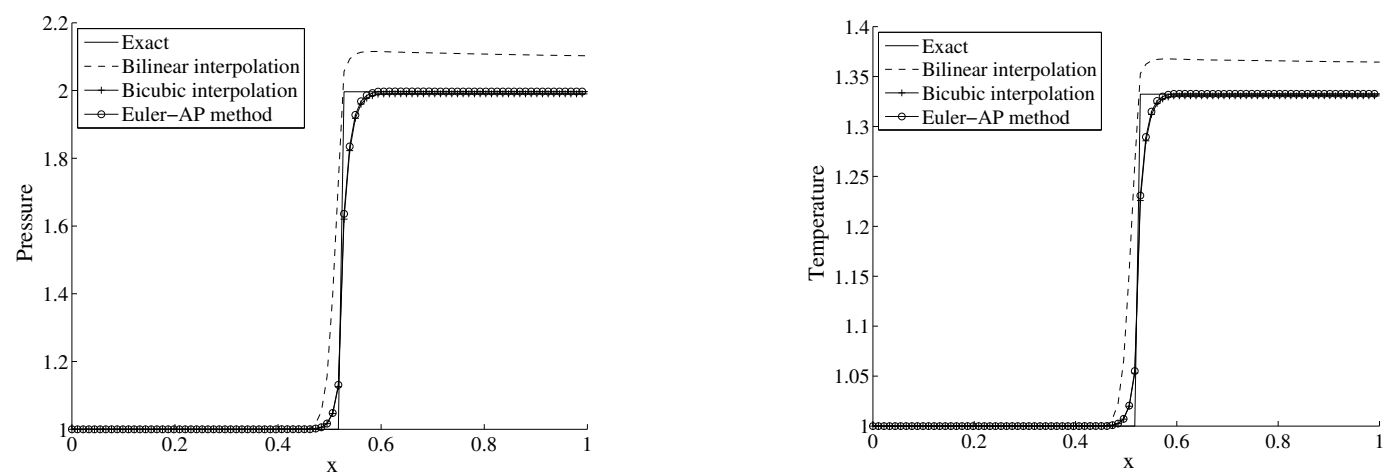

Figure 9: Comparison of the specular and Euler-AP conditions for the pressure (right) and the temperature (left).
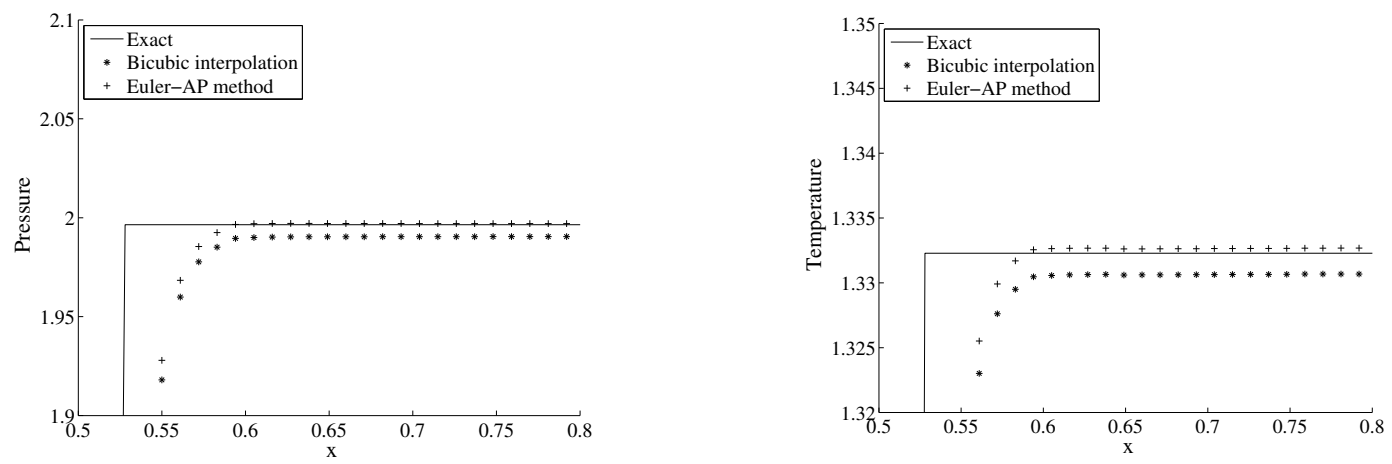

Figure 10: Zoom on post-shock values for the pressure (left) and the temperature (right).

shows that for small values of the parameter tol, the relative error on the temperature in $L_{1}$ norm is larger. It means that for small values of tol, the classical specular reflection takes over in the boundary condition $(\beta \simeq 1)$ and induces the spurious layer. Conversely, for larger values of $t o l$, we get the correct solution at the boundary (only the discretization error is left). For the first velocity grid tested $(21 \times 21)$, we have $\Delta \xi_{u}^{2} \simeq 1$ and the correct solution is obtained for tol $>10^{-1}$. The second velocity grid corresponds to $\Delta \xi_{u}^{2} \simeq 10^{-1}$ and the correct solution is 
obtained for $t o l>10^{-2}$. Thus, setting the parameter tol as $\Delta \xi_{u}^{2}$ gives the correct behaviour of the boundary condition. Moreover, the solution has a low sensibility to the value of the parameter tol as long as it is not too small meaning not lower than, approximatively, $\Delta \xi_{u}^{2} / 10$.

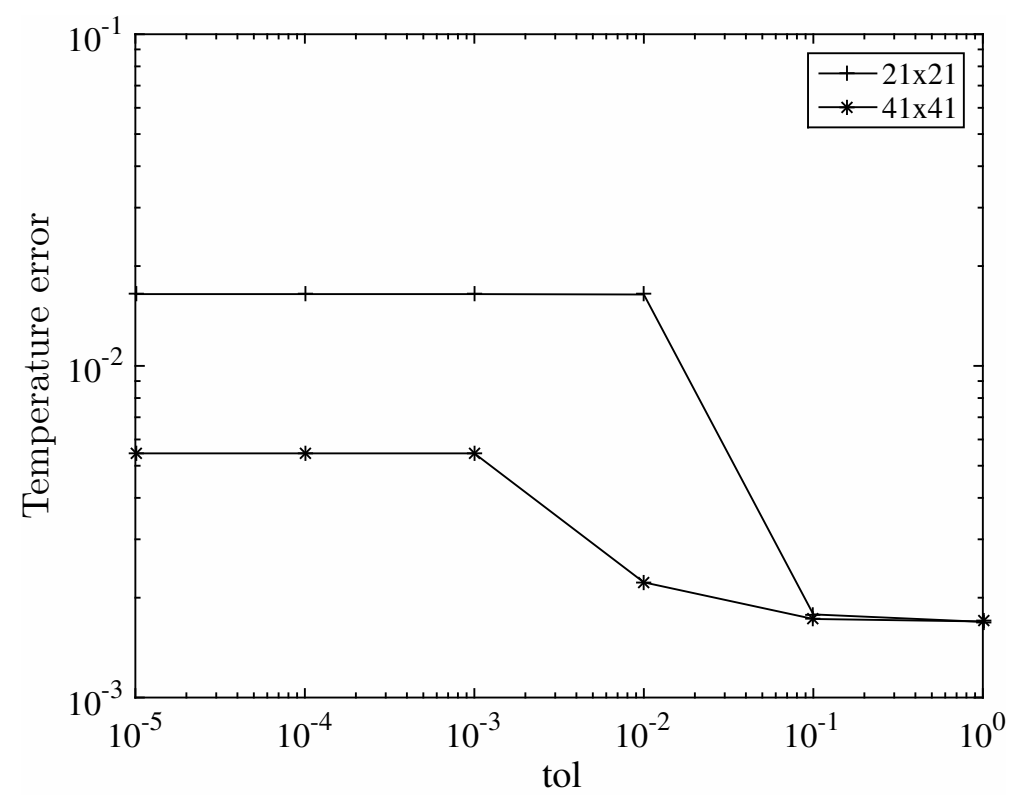

Figure 11: Temperature error in $L_{1}$ norm for two different velocity grids.

In figure 11, one can also note that the error on the temperature decreases, regardless of the value of $t o l$, when $\Delta \xi_{u}$ is reduced. This is due of course to the fact that the interpolation error decreases

\subsection{The blunt body problem}

The Euler-AP method has been validated on the oblique shock test case for a body fitted grid. In this section accuracy of the specular reflection with bicubic interpolation and Euler-AP method with $(\alpha, \beta)=0$ are compared on a Cartesian grid. A cylinder is immersed in a flow at $M=3.09(u=4, v=0, p=1, T=1$ in dimensionless form $)$. The domain is $[-0.3,0] \times[0,0.45]$ and the cylinder radius is 0.1 centred in $[0,0]$.

The impermeability condition is first computed with a purely specular reflection. The velocity grid goes from -10 to 10 in each direction and $K n_{\infty}=10^{-8}$. The boundary conditions at the border of the domain are free flow on north and east, inlet with the initial condition on west and specular reflection on south. Since the velocity grid is Cartesian, imposing the specular reflection on south can be done exactly. The impermeability condition is applied on the body.

The present method and the specular reflection with a bicubic interpolation are tested on a spatial grid $80 \times 120$ and a velocity grid $31 \times 31$. Figure 6.3 shows the solution with the Euler-AP method on the left and the normalized difference in the density between the two methods on the right.

One can observe that the difference is mostly located on the shock and on the body. The relative error is about $10 \%$ of the solution.

The same test case is solved with Euler equations. BGK simulations are compared to this Euler solution with emphasis on the boundary. The structure of the error is very close in the two cases. The largest difference is located on the shock because the solution of the BGK equation 

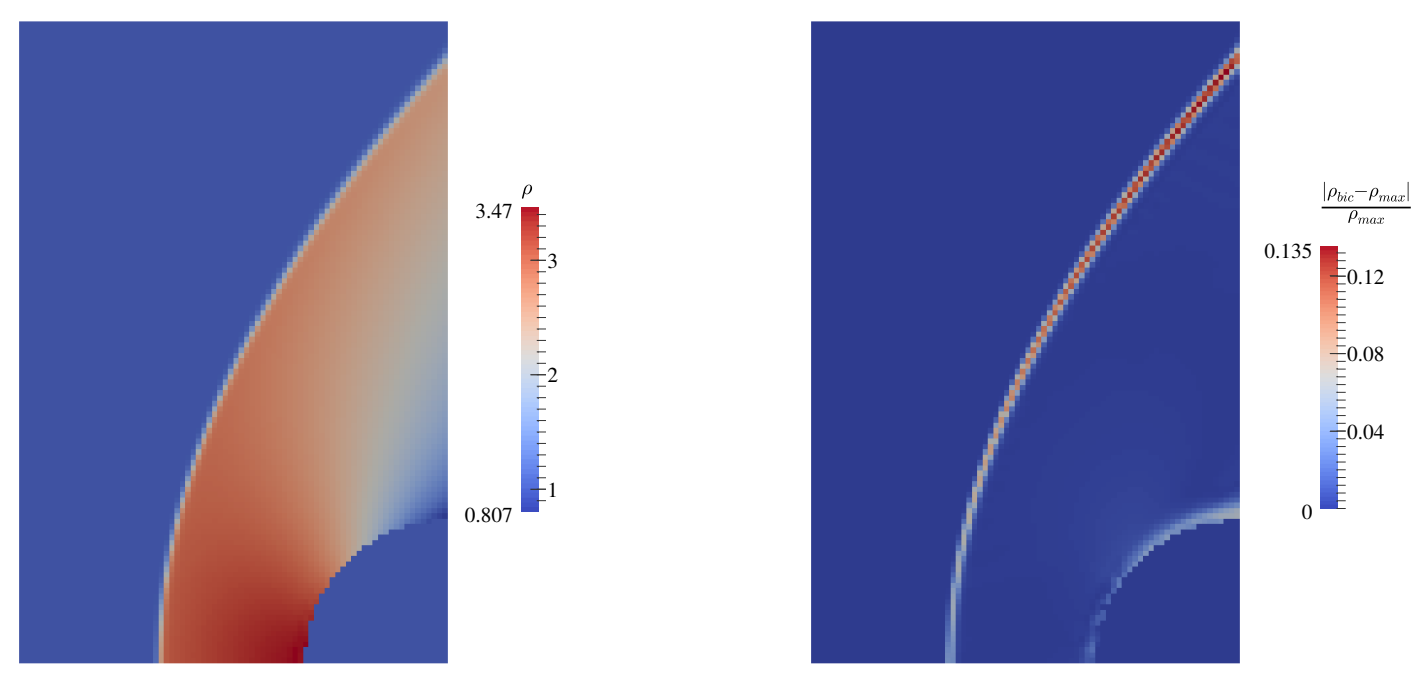

Figure 12: Left: Solution for the density with the Euler-AP method. Right: map of the difference between the two methods

is more diffusive than the one calculated with Euler equations. Thus, the shock is less sharp. On the cylinder, the error is much smaller. For the bicubic interpolation, the largest difference is about $10 \%$ while for the present method it is less than $5 \%$ of the Euler solution. As in the oblique shock case, the Euler-AP method is more accurate on the boundary (see top of the cylinder in fig. 13). It correctly preserves the asymptotic properties towards Euler equations even on Cartesian grid with immersed boundaries.
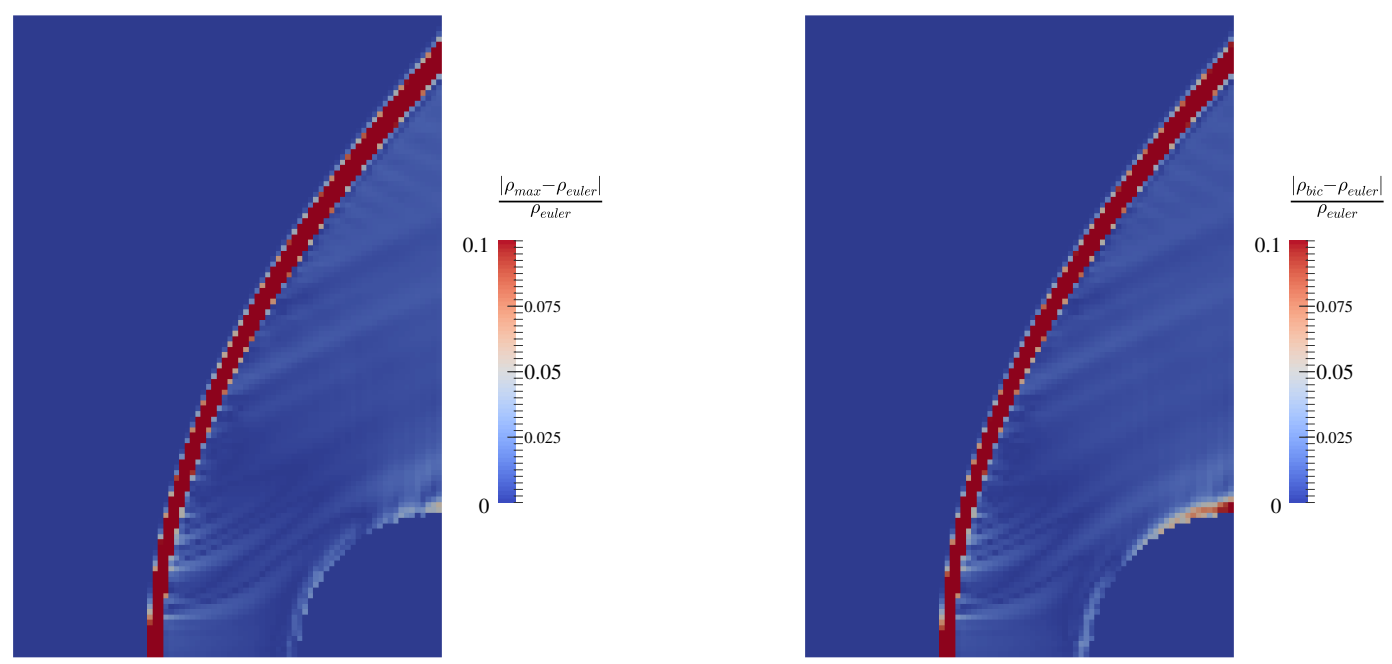

Figure 13: Error with respect to Euler solution for the Euler-AP method (left) and the bicubic interpolation (right).

Moreover the computational time is dramatically different ( 9 hours for the Euler-AP method and 19 hours for the bicubic interpolation with 128 processors).

A quantitative comparison of the computational time for the two methods is performed on 64 processors and is shown in figure 14. The Euler-AP method is clearly faster than the specular reflection with a bicubic interpolation. Also, the computational time increases faster for the bicubic interpolation as the number of velocity grid points increases (linear regression of the 


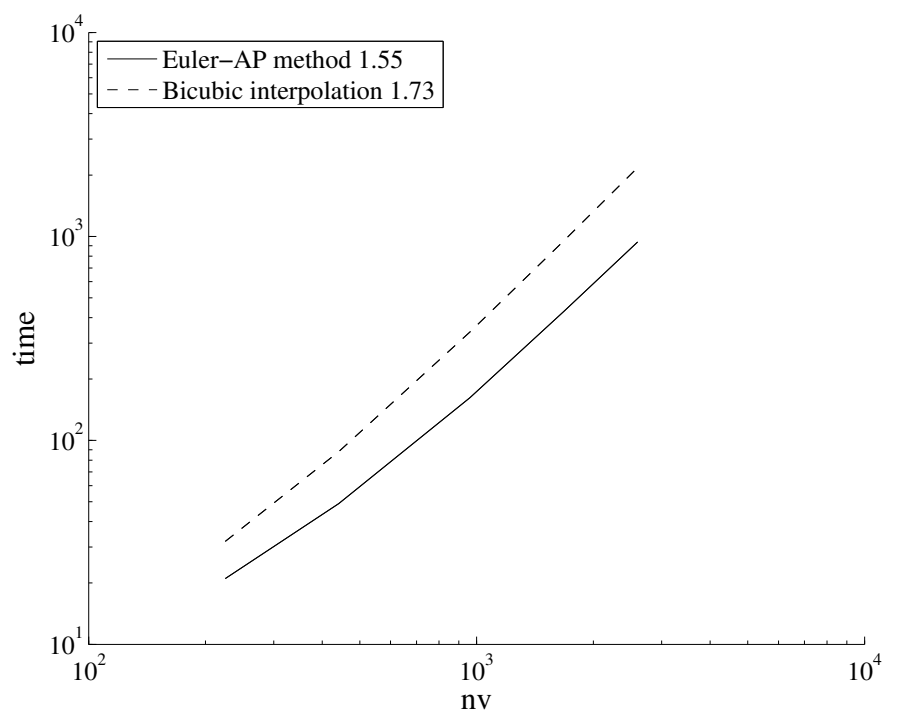

Figure 14: Computational time for the two methods with respect to the number of velocity grid points.

two curves: 1.73 against 1.55 for the present method).

\subsection{Ringleb flow}

Now that we have validated the boundary condition on body fitted grids and Cartesian grids, we show that our Cartesian method with this boundary condition is second-order accurate. Ringleb flow is a 2D steady state test case where the analytical solution can be calculated for Euler equations and will be used as a reference solution. This is a potential flow and the exact solution is obtained with the hodograph method [39].

Setting $(\theta, V)$ such that $u=V \cos \theta$ and $v=V \sin \theta$, the stream function is given by $\Psi=\frac{\sin \theta}{V}$.

The streamline equations are:

$$
x=\frac{1}{2 \rho}\left(\frac{1}{V^{2}}-2 \Psi^{2}\right)+\frac{L}{2} \quad \text { and } \quad y=\frac{\sin \theta \cos \theta}{\rho V^{2}}
$$

with (for $\gamma=5 / 3$, monoatomic gas):

$$
L=\ln \left(\frac{3 V}{6+2 \sqrt{9-3 V^{2}}}\right)-\frac{\sqrt{9-3 V^{2}}\left(V^{2}-4\right)}{V^{2}-3}, \quad c^{2}=1-\frac{\gamma-1}{2} V^{2}, \quad \rho=c^{\frac{2}{\gamma-1}}
$$

The computational domain is $[-0.5,-0.1] \times[-0.6,0]$ and the flow is solved between 2 streamlines : $\Psi_{1}=0.8$ and $\Psi_{2}=0.9$. Since $\mathbf{U} \cdot \mathbf{n}=0$ on a streamline (with $\mathbf{n}$ the normal to the streamline), any streamline can be considered as a solid boundary where the Euler impermeability condition is enforced. Here, only $\alpha$ is set as zero. The boundary conditions in inlet ( $\mathrm{y}=-0.6)$ and outlet $(\mathrm{y}=0)$ are supersonic and exactly imposed.

The finest grid used is $128 \times 192$ in space and $241 \times 241$ in velocity. To compute the space order of convergence, the solution is calculated on five different grids in space $(8 \times 12,16 \times 24$, $32 \times 48,64 \times 96$ ) but the velocity grid is kept $241 \times 241$. Thus, the result is not perturbed by convergence in velocity space. 

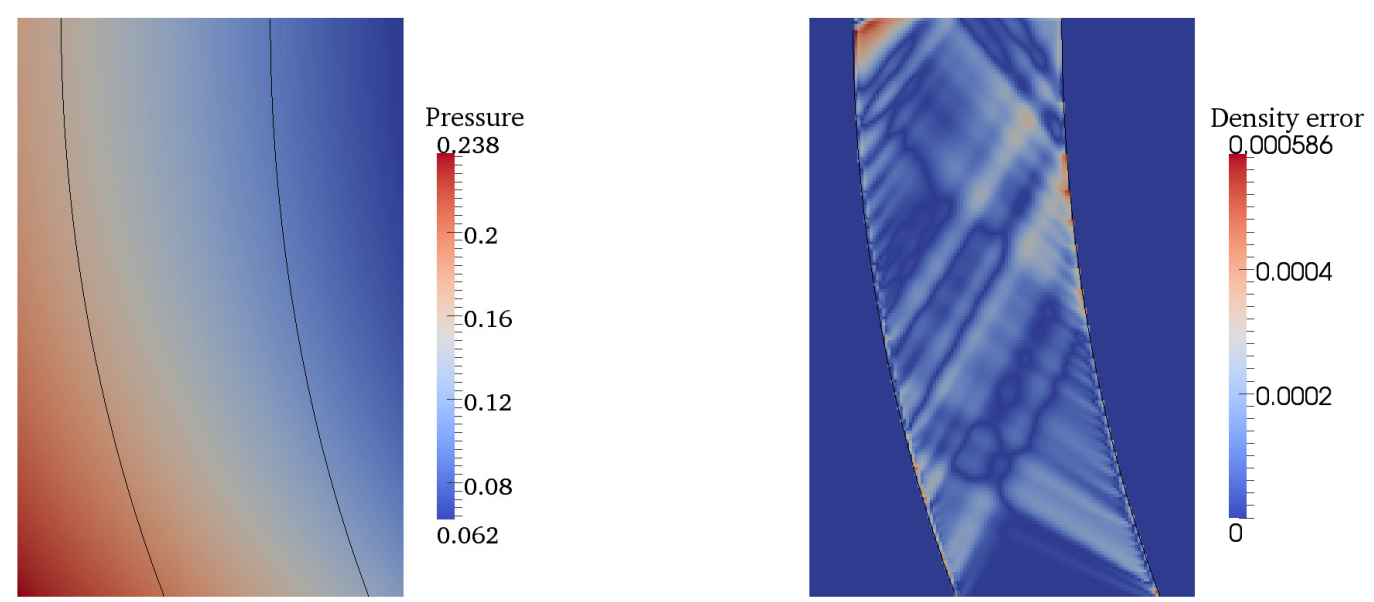

Figure 15: Left: Solution on a grid $128 \times 192$ for the pressure. Right: Error map for the density on a grid $128 \times 192$.

Figure 15 shows the solution for the pressure on the left and the error map for the density on the right. The solid black lines are the two streamlines between which the BGK equation is solved. Outside, the exact Euler solution is shown. The flow is not perturbed by the boundary condition along the streamlines and the error map on the right shows that the error with respect to the analytical solution is less than $1 \%$.
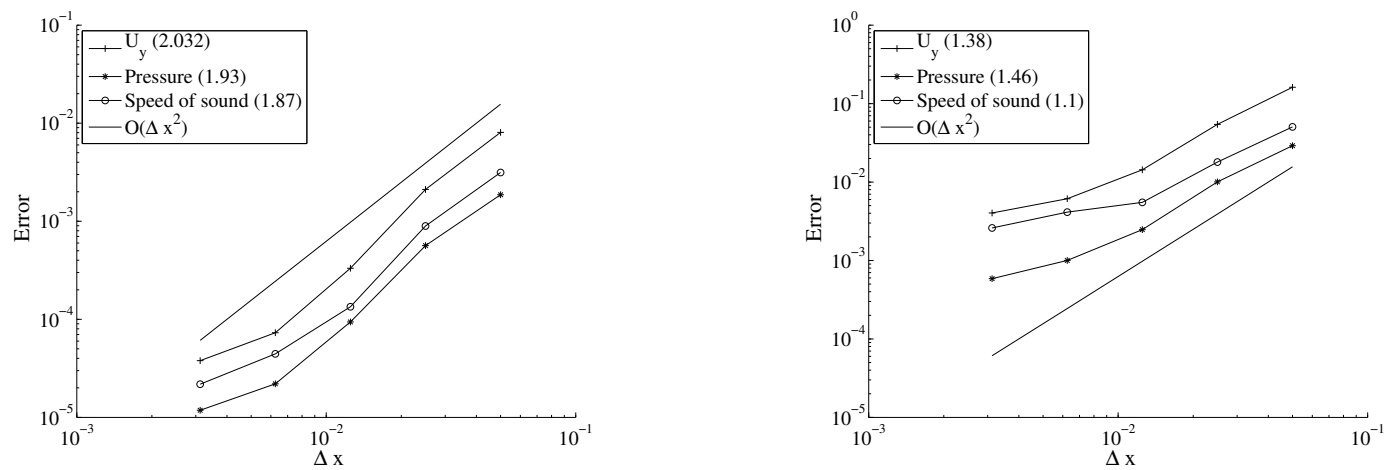

Figure 16: Error in $L_{1}$ (left) and $L_{\infty}$ norm (right).

Figure 16 shows the error with respect to the analytical solution for $L_{1}$ and $L_{\infty}$ norms (see also tables 1 and 2). Second order is obtained in $L_{1}$ norm. However, on the finest grid, it is possible to notice a slight deterioration in accuracy with respect to previous grids which actually show a convergence order larger than 2 . This phenomenon is probably due to the fact that at this degree of refinement the space error is of the same order of magnitude of the error in velocity space, where the reconstruction of the distribution function at the boundary is indeed carried out with first order accuracy. In $L_{\infty}$ norm the order is smaller (1.5 for the pressure) but it occurs on a tiny subdomain and it does not spoil the $L_{1}$ norm order of convergence.

Finally, figure 17 shows the convergence history as the velocity grid is refined (see also table 3 ). We cannot consider the analytical solution as a reference solution since the space grid is taken fixed. Reducing the error discretization in space would require a very fine grid in space making the computations prohibitive. Thus, the error is computed as the relative error on the 


\begin{tabular}{|c|c|c|c|c|c|c|}
\hline$N_{x}$ & $U_{y}$ & order & $P$ & order & Speed of sound & order \\
\hline 8 & $8.1 \times 10^{-3}$ & & $1.9 \times 10^{-3}$ & & $3.1 \times 10^{-3}$ & \\
\hline 16 & $2.1 \times 10^{-3}$ & 1.93 & $5.64 \times 10^{-4}$ & 1.725 & $8.94 \times 10^{-4}$ & 1.81 \\
\hline 32 & $3.32 \times 10^{-4}$ & 2.67 & $9.39 \times 10^{-5}$ & 2.59 & $1.34 \times 10^{-4}$ & 2.73 \\
\hline 64 & $7.29 \times 10^{-5}$ & 2.19 & $2.2 \times 10^{-5}$ & 2.1 & $4.45 \times 10^{-5}$ & 1.59 \\
\hline 128 & $3.78 \times 10^{-5}$ & 0.94 & $1.18 \times 10^{-5}$ & 0.895 & $2.18 \times 10^{-5}$ & 1.03 \\
\hline
\end{tabular}

Table 1: Error in $L_{1}$ norm.

\begin{tabular}{|c|c|c|c|c|c|c|}
\hline$N_{x}$ & $U_{y}$ & order & $P$ & order & Speed of sound & order \\
\hline 8 & 0.16 & & $2.9 \times 10^{-2}$ & & $5.04 \times 10^{-2}$ & \\
\hline 16 & $5.43 \times 10^{-2}$ & 1.57 & $1 \times 10^{-2}$ & 1.53 & $1.79 \times 10^{-2}$ & 1.49 \\
\hline 32 & $1.43 \times 10^{-2}$ & 1.93 & $2.5 \times 10^{-3}$ & 2.02 & $5.5 \times 10^{-3}$ & 1.7 \\
\hline 64 & $6.1 \times 10^{-3}$ & 1.22 & $1 \times 10^{-3}$ & 1.3 & $4.1 \times 10^{-3}$ & 0.41 \\
\hline 128 & $4 \times 10^{-3}$ & 0.6 & $5.86 \times 10^{-4}$ & 0.77 & $2.6 \times 10^{-3}$ & 0.67 \\
\hline
\end{tabular}

Table 2: Error in $L_{\infty}$ norm.

pressure between two successive grids. The space grid is kept constant $(64 \times 96)$. Hence, the convergence is not biased by the error in space. Five different velocity grids are tested: $15 \times 15$, $31 \times 31,61 \times 61,121 \times 121,241 \times 241$ giving four points on the convergence curve. It shows that for large $\Delta \xi$ the error decreases fast and then the solution converges with a second order accuracy even in $L_{\infty}$ norm (which is the accuracy of the quadrature rule).

\subsection{Flow past a vertical plate}

Several test cases have been so far presented to show the accuracy of the Euler-AP boundary condition in the continuum regime, for body-fitted and Cartesian meshes. We now validate the Euler-AP wall condition in the rarefied regime.

The flow past a vertical plate was first introduced by Bird [6] to study the formation of vortices at different Knudsen numbers. This case highlights the rarefied effects for a simple geometry, with a specular boundary condition applied on the vertical plate. Our results are compared to the numerical ones obtained by Bird [6] with DSMC and by Chen et al. [9] with AUGKS.

A vertical plate is immersed in a flow at Mach 0.53 of Argon. The undisturbed initial flow is imposed at inlet $(x=-4 / 3)$, outlet $(x=2)$, at the upper boundary $(y=2), u_{\infty}=0.69$ and a temperature of 1 (in dimensionless values). At $y=0$, a symmetric boundary condition is enforced corresponding to the specular reflection. The plate is placed at $x=0$ and goes from $y=0$ to $y=1$ (height $\mathrm{h}=1$ ). It has zero thickness and is situated exactly at the interface between two cells. The physical space is discretized with $100 \times 60$ cells while the velocity space has 41 cells in each directions. The Euler-AP condition is imposed on both sides of the plate

\begin{tabular}{|c|c|c|c|c|}
\hline$N_{v x}$ & $L_{1}$ & order & $L_{\infty}$ & order \\
\hline 15 & 0.05 & & 0.0604 & \\
\hline 31 & $3.46 \times 10^{-6}$ & 10.01 & $9.35 \times 10^{-5}$ & 8.91 \\
\hline 61 & $1.82 \times 10^{-6}$ & 0.9491 & $2.32 \times 10^{-5}$ & 2.06 \\
\hline 121 & $4.3 \times 10^{-7}$ & 2.1065 & $5.1 \times 10^{-6}$ & 2.21 \\
\hline
\end{tabular}

Table 3: $L_{1}$ and $L_{\infty}$ norm of the pressure error for the Ringleb flow on the $64 \times 96$ space grid. 


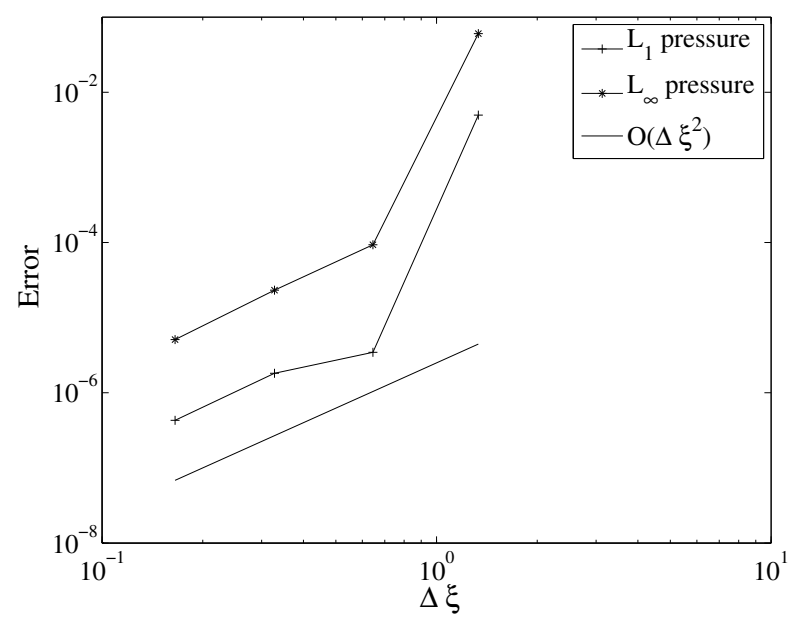

Figure 17: $L_{1}$ and $L_{\infty}$ norm of the pressure error for the Ringleb flow on the $64 \times 96$ space grid.

with our second order scheme.

The steady state of this problem shows a vortex behind the plate. As mentioned by Bird, the solution strongly depends on the boundaries of the domain. However, for the same computational domain and boundary conditions, comparisons can be made with the results obtained by Bird on the velocity profile along a vertical axis passing through the center of the vortex for different Knudsen numbers. We note that for this test case, the results are presented with the value of the Knudsen number in Bird's sense $\left(K n_{B}\right)$. To fit with our model, we actually take $K n_{\infty}=K n_{B} \frac{5 \sqrt{2 \pi}}{16}$.
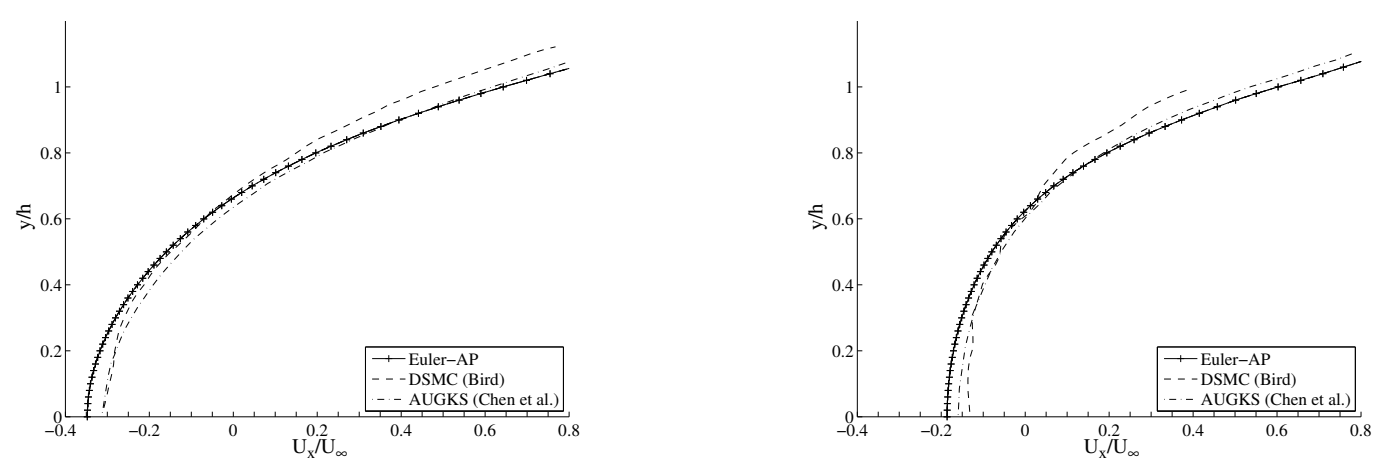

Figure 18: Left: $K n_{B}=0.044$. Right: $K n_{B}=0.088$.

Figures 18 and 19 show that the velocity profiles obtained with the Euler-AP boundary condition and a second-order scheme are in good agreement with Bird's results for different Knudsen numbers. The results show that the vortex becomes stronger as the Knudsen number decreases. Small variations of the position of the vortex center definition induce large variations of the velocity at $y=0$. In this case, the value of the parameter $\beta$ is 0 at the initial state but goes quickly towards 1 for all boundary points since the Knudsen number is large. 

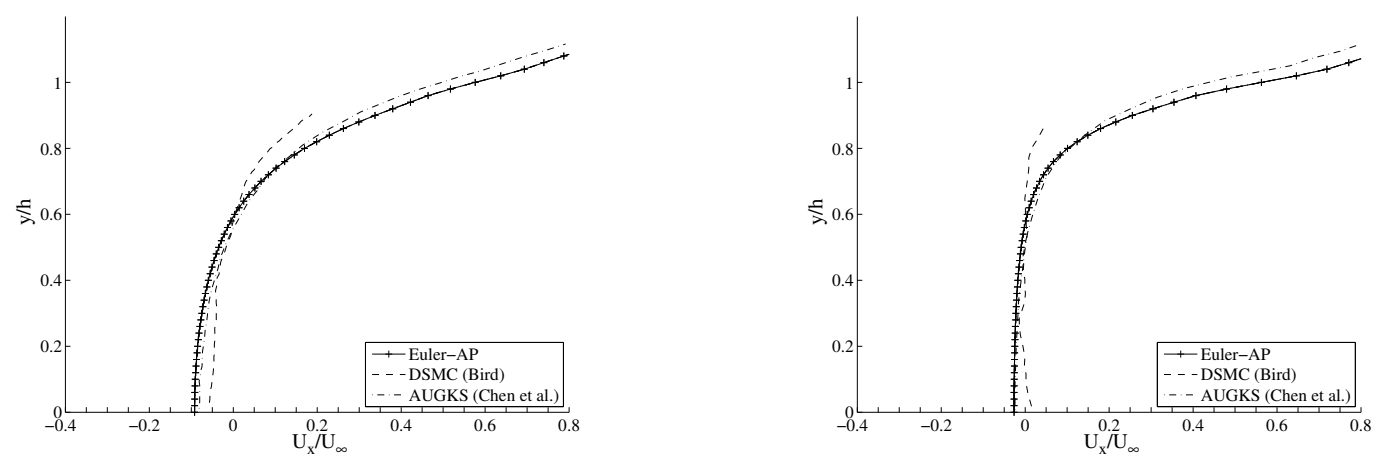

Figure 19: Left: $K n_{B}=0.147$. Right: $K n_{B}=0.293$.

\subsection{A nozzle plume}

We consider a final qualitative validation against experimental results. A flow expands at the outlet of a nozzle in a low pressure atmosphere. The spreading jet has been studied for example by Latvala et al. in [29] and [30]. Experiments were performed for different ambient pressures to determine the angle and the shape of the jet at the outlet of the nozzle.

The area ratio between the throat and the outlet of the nozzle is 4.8. Here, we impose the total pressure $\left(P_{t o t}=1\right)$ and the total temperature $\left(T_{t o t}=0.6\right)$ at the inlet of the nozzle. Considering that Mach 1 is reached at the throat, one can determine the Mach number, the pressure and the temperature at the outlet under the assumption of a one dimensional isentropic flow. In our case, this gives $\mathrm{M}=3.7763, \mathrm{~T}=0.1738$ and $\mathrm{p}=0.0126$. This pressure is called the adaptation pressure $P_{c}$. If the ambient pressure $P_{a t m}$ is lower than $P_{c}$ the flow inside the nozzle is independent of $P_{a t m}$.

We obtain the jet angle by tracking the contact discontinuity between the gas coming from the nozzle and the gas initially outside the nozzle with a level set function keeping fixed the point at the extremity of the nozzle $(x=2)$. At each time step, the level set function is transported according to the velocity of the fluid with the procedure described in section 4 for moving boundaries. In the cells initially outside the fluid domain and freshly appeared (determined by the cell center) in the jet, the computed boundary condition is imposed. All the viscous effects (mixing layer) are supposed to be concentrated across the contact discontinuity represented by the isoline zero of the level set function. The velocity of the contact discontinuity is computed thanks to a Riemann problem where only the ambient pressure $P_{a t m}$ is imposed.

At initial state, the nozzle is filled with a gas at rest with $p=1$ and $T=0.6$. Outside of the nozzle, the gas is also at rest, with $p=P_{a t m}$ and $T=0.6$. The initial configuration with the zero isoline of the level set function is shown on figure 20. The dark area above the nozzle is penalized, no computations are performed unless the level set evolves and includes this region. The computational domain is $[-1,5] \times[0,4]$ and is discretized with $120 \times 80$ cells. The velocity space $[-10,10] \times[-10,10]$ is discretized with 101 points in each directions.

The solution for $P_{c} / P_{a t m}=2000$ is shown on figures $21,22,23$ and 24 for different times. First, the flow goes out of the nozzle and turns back because of the abrupt expansion $(t=1.2$ and $t=5)$. Then, when the flows stabilizes in the nozzle, a shock propagates from the inlet towards the outlet. On figure 23 , at $t=11.1$ the shock is at $x=2.7$ and establishes the angle of the jet with the nozzle outlet.

Figure 25 shows the angle $\delta$ of the jet at the outlet for different pressure ratio $P_{c} / P_{a t m}$ and for BGK and compressible Euler models. When this ratio is equal to 1, the angle of the flux at the outlet should be the same as the angle of the nozzle outlet. The figure shows that in this 


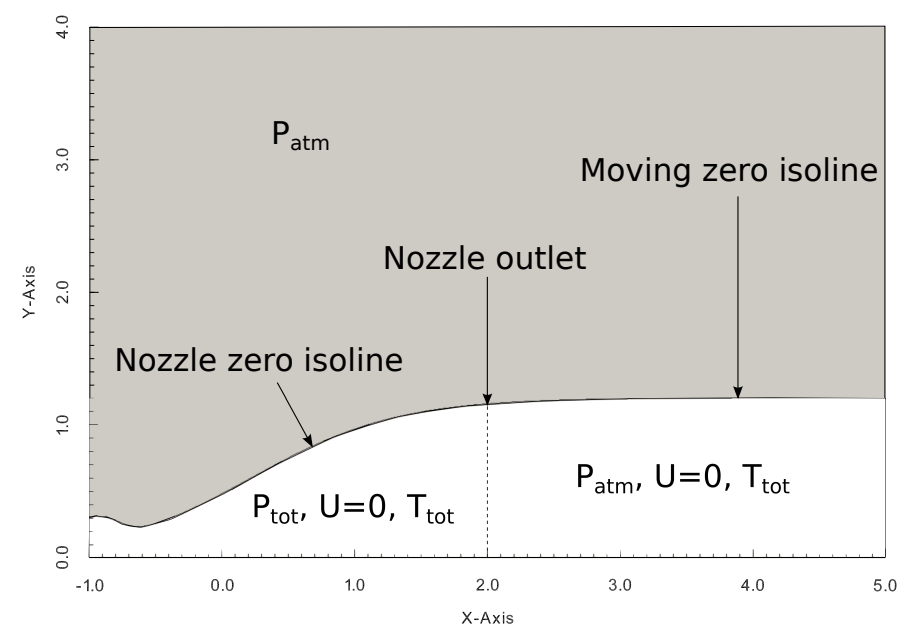

Figure 20: Computational domain at $\mathrm{t}=0$.

Time: 1.2171005

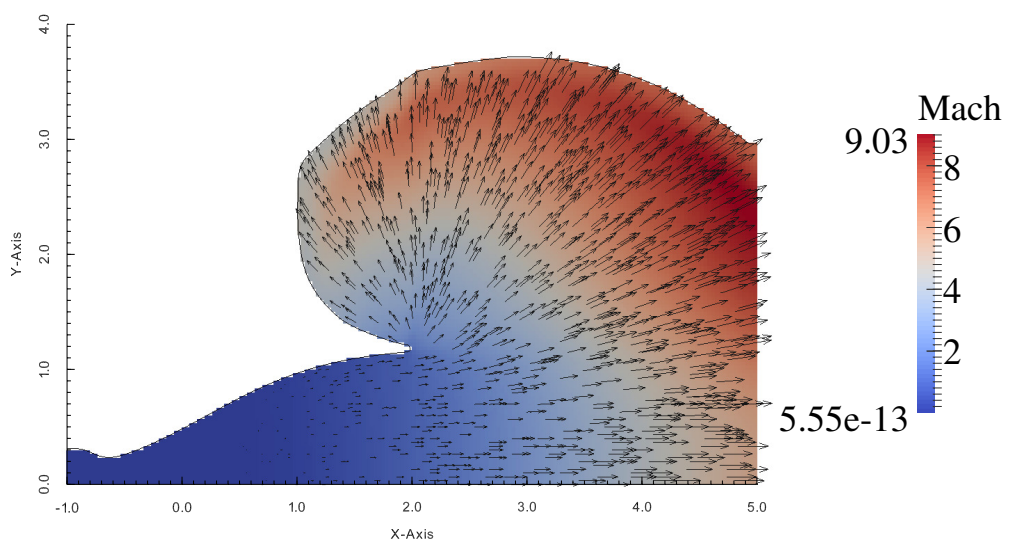

Figure 21: Mach number and velocity vectors at $\mathrm{t}=1.2$ for $P_{c} / P_{a t m}=2000$.

Time: 5.050505

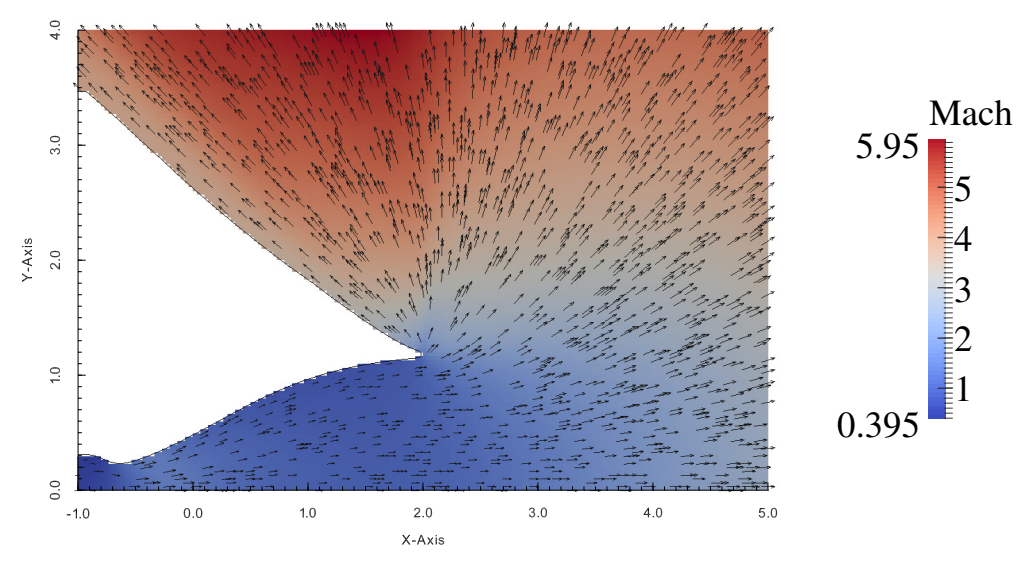

Figure 22: Mach number and velocity vectors at $\mathrm{t}=5$ for $P_{c} / P_{a t m}=2000$. 
Time: 11.110505

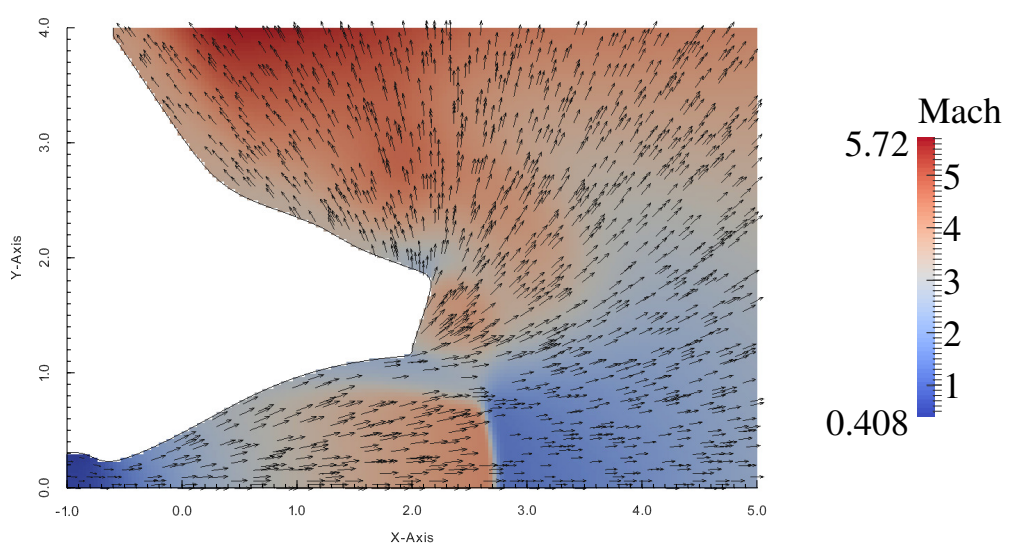

Figure 23: Mach number and velocity vectors at $\mathrm{t}=11$ for $P_{c} / P_{a t m}=2000$.

Time: 15.137375

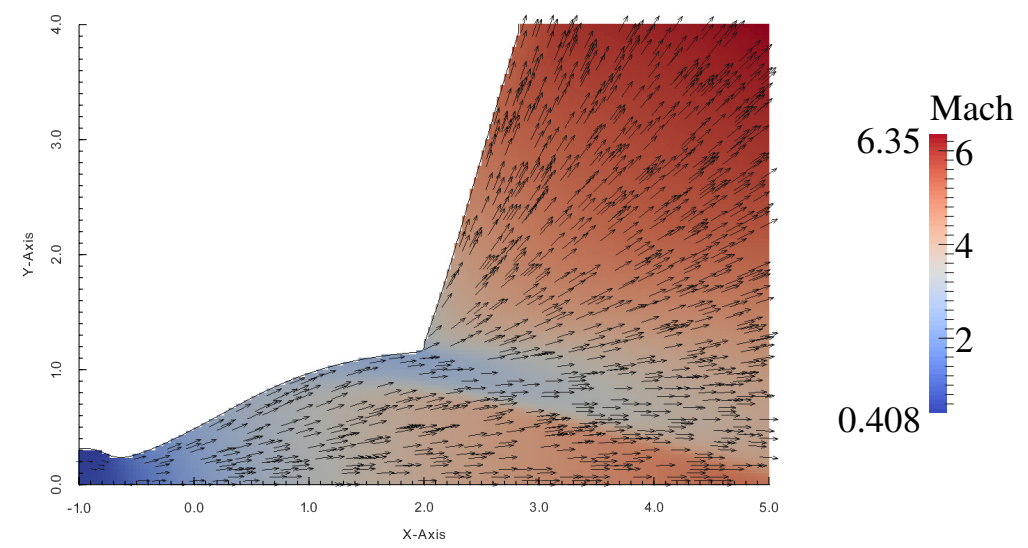

Figure 24: Mach number and velocity vectors at steady state for $P_{c} / P_{a t m}=2000$. 


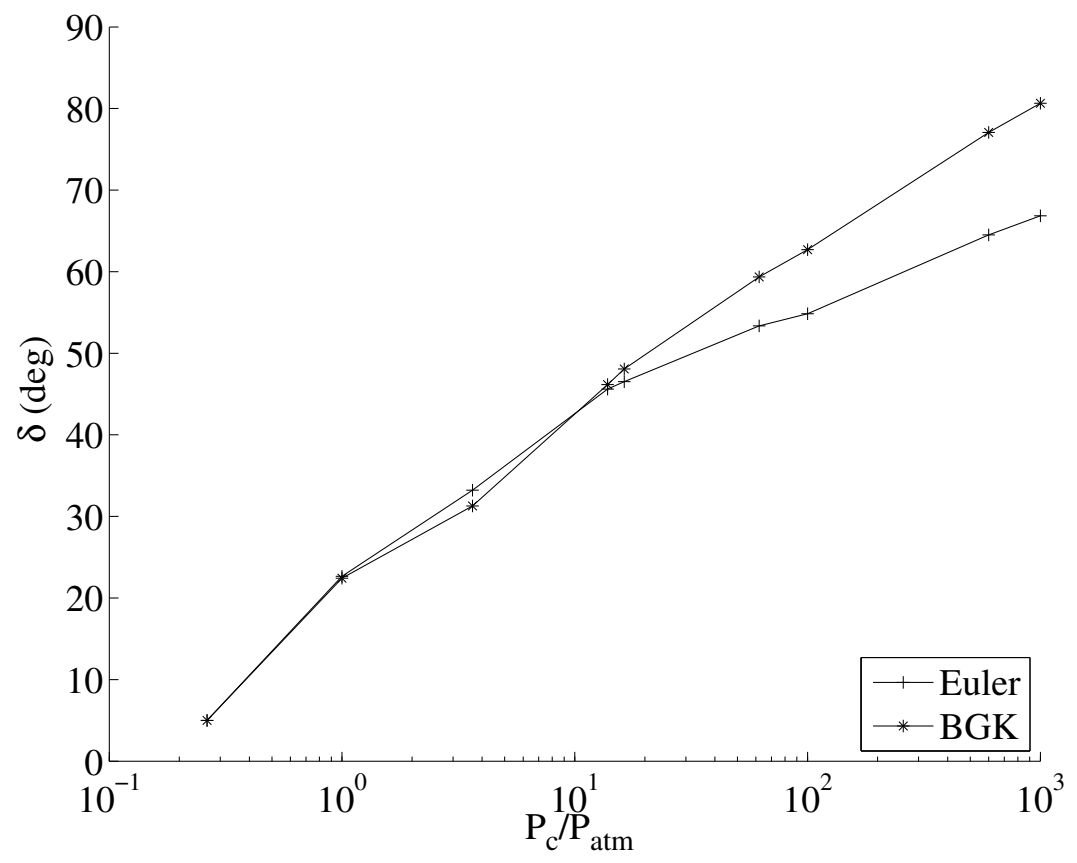

Figure 25: Angle for different pressure ratios calculated with Euler and BGK models.

configuration, $\delta$ is actually larger. This is due to the calculation of $P_{c}$ through $1 \mathrm{D}$ relations. Thus, the pressure at the boundary of the nozzle at the outlet is not equal to $P_{c}$ but it is larger. If this pressure is imposed outside the nozzle, we obtain an angle $\delta$ equal to the one of the nozzle exit (first point on figure 25). These results can be qualitatively compared with the experimental results obtained by Latvala et al. in [29] where it is shown, for $\gamma=7 / 5$, that the evolution of the jet angle is linear with the logarithm of the pressure ratio. The same behaviour is observed in figure 25 for the BGK model. The compressible Euler model progressively detaches from a straight line as the rarefaction increases. The quantitative results cannot be directly compared to experiments since in our case, since $\gamma=5 / 3$.

For small pressure ratios $(<10)$ the BGK and compressible Euler models give the same angle. When the ratio increases, the difference becomes larger and the kinetic model stays very close to a straight line. For this kind of pressure ratio, the local relaxation time increases outside the nozzle and becomes too large to consider the fluid at equilibrium. Thus, the continuum model tends to give a different solution. This emphasizes the necessity of using a kinetic model with an AP boundary condition since this problem cannot be solved with a continuum model for high pressure ratio. Also, a solution computed with a specular reflection wall condition in the nozzle would significantly pollute the simulation because of a net reduction of the efficient section of the nozzle.

\section{Conclusion}

In recent years, the notion of Asymptotic Preserving schemes [17] has been introduced answering the need for numerical methods that automatically converge to discretizations of appropriate reduced models, as the Knudsen number changes within the flow. This work shows that such methods are not AP in presence of interior boundaries, because they exhibit spurious energy fluxes through the boundaries, when the hydrodynamic limit is approached. These artefacts are purely numerical, and a possible cure relies on a more accurate treatment of the boundary 
condition, at the price of costly higher order interpolations in velocity space. Here we propose an efficient boundary condition which ensures that an AP scheme remains AP up to the boundary. We illustrate this result by comparing several numerical schemes to model the impermeability condition for the BGK model with emphasis on asymptotic preserving properties in the Euler limit. Moreover, we also propose a technique to impose the boundary conditions on Cartesian grids, specializing the method of immersed boundaries to the case of kinetic fluid flows. We have also shown how to recover second order accuracy on Cartesian meshes using this new wall condition and simulated non-trivial rarefied regime test cases. In particular, the last test case highlights the necessity of a kinetic model with the correct asymptotic behaviour. Such cases cannot be correctly simulated with a continuous model. In future work, we will concentrate on the asymptotic limit of wall models towards Navier-Stokes equations using an ES-BGK model.

\section{Acknowledgements}

Experiments presented in this paper were carried out using the PlaFRIM experimental testbed, being developed under the Inria PlaFRIM development action with support from LABRI and IMB and other entities: Conseil Régional d'Aquitaine, FeDER, Université de Bordeaux and CNRS (see https://plafrim.bordeaux.inria.fr/).

This study has been carried out with financial support from the French State, managed by the French National Research Agency (ANR) in the frame of the "Investments for the future" Programme IdEx Bordeaux (ANR-10-IDEX-03-02), Cluster of excellence CPU.

Gabriella PUPPO acknowledges the contribution of PRIN project 2009, number 2009588FHJ_002.

\section{Appendix}

We present the Newton-Raphson algorithm to compute the discrete Maxwellian distribution function. The first three moments of the distribution functions are known and we define the collision invariant $\mathbf{m}(\boldsymbol{\xi})=\left(1, \boldsymbol{\xi}, \frac{1}{2}|\boldsymbol{\xi}|^{2}\right)^{T}$. The Maxwellian distribution function $M_{f}$ is computed such that, with the given quadrature rule:

$$
\left\langle M_{f}, \mathbf{m}(\boldsymbol{\xi})\right\rangle=\left(\begin{array}{c}
\rho \\
\rho \mathbf{U} \\
E
\end{array}\right)
$$

where $(\rho, \rho \mathbf{U}, E)=\langle f, \mathbf{m}(f)\rangle$, that is the moments are computed from $f$ approximately, using the given quadrature rule.

We are looking for $M_{f}$ under the form: $M_{f}=\exp (\boldsymbol{\alpha} \cdot \mathbf{m}(\boldsymbol{\xi}))$. Let us define the function:

$$
\mathbf{F}(\boldsymbol{\alpha})=\langle\exp (\boldsymbol{\alpha} \cdot \mathbf{m}(\boldsymbol{\xi})), \mathbf{m}(\boldsymbol{\xi})\rangle-\underline{\boldsymbol{\rho}}
$$

with $\boldsymbol{\rho}=(\rho, \mathbf{U}, E)^{T}$, the macroscopic variables computed from the moments of $f$. The discrete Maxwellian verifies $\mathbf{F}(\boldsymbol{\alpha})=0$. Thus, the problem of computing the discrete Maxwellian reduces to find $\boldsymbol{\alpha}$ such that $\mathbf{F}(\boldsymbol{\alpha})=0$. This is done with a Newton-Raphson algorithm. The initial value of $\boldsymbol{\alpha}$ corresponds to the continuous Maxwellian:

$$
\boldsymbol{\alpha}_{c}=\left(\ln \left(\frac{\rho}{(2 \pi T)^{N / 2}}\right)-\frac{|\mathbf{U}|^{2}}{2 T}, \frac{\mathbf{U}}{T},-\frac{1}{T}\right)
$$

The algorithm is summarized as follows: 
$\star \alpha=\alpha_{c}$

$\star$ Do while $\left(\mathbf{F}(\boldsymbol{\alpha})>10^{-6}\right)$

Compute $\operatorname{Jac}(\mathbf{F})=\frac{\partial F_{i}}{\partial \alpha_{j}}$ for $i, j=1, \ldots d+2$

Compute $\mathbf{F}(\boldsymbol{\alpha})$

$\boldsymbol{\alpha}=\boldsymbol{\alpha}-J a c(\mathbf{F}) \cdot \mathbf{F}(\boldsymbol{\alpha})$

$\star$ Compute $M_{f}=\exp (\boldsymbol{\alpha} \cdot \mathbf{m}(\boldsymbol{\xi}))$

In the case of the reduced models, the algorithm is identical but the function $\mathbf{F}$ is defined as:

$$
\begin{aligned}
& \mathbf{F}(\boldsymbol{\alpha})=\left\langle\widetilde{M_{\phi}}, \mathbf{m}_{1}(\boldsymbol{\xi})\right\rangle+\left\langle\widetilde{M_{\psi}}, \mathbf{e}_{3}\right\rangle-\left\langle f, \mathbf{m}_{1}(\boldsymbol{\xi})\right\rangle \text { in 1D } \\
& \mathbf{F}(\boldsymbol{\alpha})=\left\langle\widetilde{M_{\phi}}, \mathbf{m}_{2}(\boldsymbol{\xi})\right\rangle+\left\langle\widetilde{M_{\psi}}, \mathbf{e}_{4}\right\rangle-\left\langle f, \mathbf{m}_{2}(\boldsymbol{\xi})\right\rangle \text { in 2D }
\end{aligned}
$$

where $\mathbf{e}_{3}=(0,0,1)^{T}, \mathbf{e}_{4}=(0,0,0,1)^{T}, \mathbf{m}_{1}(\boldsymbol{\xi})=\left(1, \xi_{u}, \frac{1}{2}\left|\xi_{u}\right|^{2}\right)^{T}, \mathbf{m}_{2}(\boldsymbol{\xi})=\left(1, \xi_{u}, \xi_{v}, \frac{1}{2}\left(\xi_{u}^{2}+\xi_{v}^{2}\right)\right)^{T}$. And:

$$
\begin{aligned}
& \boldsymbol{\alpha}_{c}=\left(\ln \left(\frac{\rho}{\sqrt{2 \pi T}}\right)-\frac{u^{2}}{2 T}, \frac{u}{T},-\frac{1}{T}\right)^{T} \text { in } 1 \mathrm{D} \\
& \boldsymbol{\alpha}_{c}=\left(\ln \left(\frac{\rho}{2 \pi T}\right)-\frac{u^{2}+v^{2}}{2 T}, \frac{u}{T}, \frac{v}{T},-\frac{1}{T}\right)^{T} \text { in } 2 \mathrm{D}
\end{aligned}
$$

The Newton-Raphson algorithm give the expression of $\widetilde{M_{\phi}} \cdot \widetilde{M_{\psi}}$ is then easily computed with the formula:

$$
\widetilde{M_{\psi}}=\frac{(N-d) T}{2} \widetilde{M_{\phi}}
$$

\section{References}

[1] Alaia, A., Puppo, G.:A Hybrid Method for Hydrodynamic-Kinetic Flow - Part II - Coupling of Hydrodynamic and Kinetic Models. Journal of Computational Physics 231(16), 5217$5242(2012)$

[2] Ascher, U.M., Ruuth, S.J., Spiteri, R.J.: Implicit-explicit Runge-Kutta methods for timedependent partial differential equations. Applied Numerical Mathematics. An IMACS Journal 25(2-3), 151-167 (1997)

[3] Bennoune, M., Lemou, M., Mieussens, L.: Uniformly stable numerical schemes for the Boltzmann equation preserving the compressible Navier-Stokes asymptotics. J. Comput. Phys. 227(8), 3781-3803 (2008)

[4] Bernard, F., Iollo, A., and Puppo, G. : A Local Velocity Grid Approach for BGK Equation. Communications in Computational Physics 16 (4), 956-982 (2014).

[5] Bhatnagar, P.L., Gross, E.P., Krook, M.: A model for collision processes in gases. I. Small amplitude processes in charged and neutral one- component systems. Phys. Rev. 94, 511-525 (1954). DOI 10.1103/PhysRev.94.511. URL http://link.aps.org/doi/10.1103/PhysRev.94.511 
[6] Bird, G.A.: Molecular Gas Dynamics and the Direct Simulation of Gas Flows. Oxford Engineering Science Series. Clarendon Press (1994). URL http://books.google.it/books?id=Bya5QgAACAAJ

[7] Cabannes, H., Gatignol, R., Luol, L.S.: The discrete Boltzmann equation. Lecture Notes at University of California, Berkley pp. 1-65 (1980)

[8] Cercignani, C.: The Boltzmann Equation and Its Applications. Springer-Verlag GmbH (1988)

[9] Chen, S., Xu, K., Lee, C., Cai, Q.: A unified gas kinetic scheme with moving mesh and velocity space adaptation. Journal of Computational Physics 231(20), 6643-6664 (2012)

[10] Chu, C.K.: Kinetic-theoretic description of the formation of a shock wave. Phys. Fluids 8, 12-22 (1965)

[11] Cisternino, M., Weynans, L.: A Parallel Second Order Cartesian Method for Elliptic Interface Problems. Communications in Computational Physics 12(5), 1562-1587 (2012). DOI 10.4208/cicp.160311.090112a

[12] Degond P., Jin S., Mieussens, L.: A smooth transition model between kinetic and hydrodynamic equations. Journal of Computational Physics 209(1), 665-694, (2005).

[13] Degond, P., Pareschi, L., Russo, G.: Modeling and Computational Methods for Kinetic Equations. Modeling and Simulation in Science, Engineering and Technology. Springer (2004)

[14] Dimarco, G., Pareschi, L.: Exponential Runge-Kutta methods for stiff kinetic equations. SIAM Journal on Numerical Analysis 49(5), 2057-2077 (2011)

[15] Fedkiw, R.P.: Coupling an Eulerian fluid calculation to a Lagrangian solid calculation with the ghost fluid method. Journal of Computational Physics 175(1), 200-224 (2002)

[16] Fedkiw, R.P., Aslam, T., Merriman, B., Osher, S.: A non-oscillatory Eulerian approach to interfaces in multimaterial flows (the ghost fluid method). Journal of Computational Physics 152(2), 457-492 (1999)

[17] Filbet, F., Jin, S.: A class of asymptotic-preserving schemes for kinetic equations and related problems with stiff sources. Journal of Computational Physics 229(20), 7625-7648 (2010)

[18] Filbet, F., Yang, C.: An inverse Lax-Wendroff method for boundary conditions applied to Boltzmann type models. Journal of Computational Physics 245, 43-61 (2013)

[19] Gibou, F., Fedkiw, R.P., Cheng, L.T., Kang, M.: A second-order-accurate symmetric discretization of the Poisson equation on irregular domains. Journal of Computational Physics 176(1), 205-227 (2002)

[20] Goodman F.O., Wachman H.Y.: Dynamics of gas-surface scattering. Academic press, New York, 1976

[21] Gorsse, Y., Iollo, A., Telib, H., Weynans, L.: A simple second order cartesian scheme for compressible Euler flows. Journal of Computational Physics 231(23), 7780-7794 (2012) 
[22] Ingram, D.M., Causon, D.M., Mingham, C.G.: Developments in Cartesian cut cell methods. Mathematics and Computers in Simulation 61(3-6), 561-572 (2003)

[23] Jiang, G.S., Shu, C.W.: Efficient Implementation of Weighted ENO Schemes. Journal of Computational Physics 126(1), 202-228 (1996)

[24] Jin, C., Xu, K.: A unified moving grid gas-kinetic method in Eulerian space for viscous flow computation. Journal of Computational Physics 222(1), 155-175 (2007)

[25] Jin, S.: Efficient asymptotic-preserving (AP) schemes for some multiscale kinetic equations. SIAM Journal on Scientific Computing 21(2), 441-454 (1999)

[26] Jin, S.: Asymptotic preserving (AP) schemes for multiscale kinetic and hyperbolic equations: a review. Rivista di Matematica della Università di Parma. Serie 7 3(2), 177-216 (2012)

[27] Karniadakis, G., Beskok, A., Aluru, N.: Microflows and nanoflows : fundamentals and simulation. Springer (2005)

[28] Kennedy, C.A., Carpenter, M.H.: Additive Runge-Kutta schemes for convection-diffusionreaction equations. Applied Numerical Mathematics 44(1-2) (2003)

[29] Latvala, E.K., Anderson, T.P.: Experimental Determination of Jet Spreading from Supersonic Nozzles at High Altitudes. AEDC-TN-58-98 (ASTIA Document No. AD-208546) (1959)

[30] Latvala, E.K., Anderson, T.P.: Studies of the Spreading of Rocket Exhaust Jets at High Altitudes. Planetary and Space Science 4, 77-91 (1961)

[31] Mieussens, L.: Discrete-Velocity Models and Numerical Schemes for the Boltzmann-BGK Equation in Plane and Axisymmetric Geometries. Journal of Computational Physics 162(2), 429-466 (2000)

[32] Osher, S., Sethian, J.A.: Fronts propagating with curvature-dependent speed : Algorithms based on hamilton jacobi formulations. Journal of Computational Physics 79(12) (1988)

[33] Pareschi, L., Russo, G.: Implicit-Explicit Runge-Kutta Schemes and Applications to Hyperbolic Systems with Relaxation. Journal of Scientific Computing 25(1), 129-155 (2005)

[34] Pareschi, L., Russo, G.: Efficient asymptotic preserving deterministic methods for the Boltzmann equation. Models and Computational Methods for Rarefied Flows, AVT- 194 RTO AVT/VKI, Rhode St. Genese, Belgium (2011)

[35] Peskin, C.S.: Flow patterns around heart valves: A numerical method. Journal of Computational Physics 10(2), 252 - 271 (1972). DOI 10.1016/0021-9991(72)90065-4. URL http://www.sciencedirect.com/science/article/pii/0021999172900654

[36] Pieraccini, S., Puppo, G.: Implicit-Explicit schemes for BGK kinetic equations. Journal of Scientific Computing 32(1), 1-28 (2007)

[37] Pieraccini, S., Puppo, G.: Microscopically Implicit-Macroscopically Explicit schemes for the BGK equation. Journal of Computational Physics 231, 299-327 (2012)

[38] Sethian, J.A.: Fast Marching Methods. SIAM Review 41, 199-235 (1999) 
[39] Shapiro, A.: The Dynamics and Thermodynamics of Compressible Fluid Flow. Ronald Press (1953)

[40] Tiwari S., Klar A.: An adaptive domain decomposition procedure for Boltzmann and Euler equations. Journal of Computational and Applied Mathematics 90, 223-237 (1998)

[41] Xu, K., Huang, J.C.: A unified gas-kinetic scheme for continuum and rarefied flows. Journal of Computational Physics 229(20), 7747-7764 (2010)

[42] Yang, G., Causon, D., Ingram, D., Saunders, R., Batten, P.: A cartesian cut cell method for compressible flows. Part A. static body problems. Aeronaut. J. pp. 47-56 (1997) 